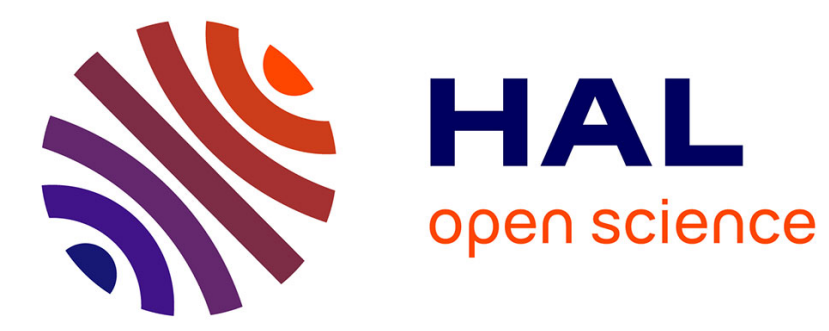

\title{
Spatio-temporal modelling of the Hes1 and p53-Mdm2 intracellular signalling pathways
}

Marc Sturrock, Alan J. Terry, Dimitris P. Xirodimas, Alastair M. Thompson, Mark A.J. Chaplain

\section{> To cite this version:}

Marc Sturrock, Alan J. Terry, Dimitris P. Xirodimas, Alastair M. Thompson, Mark A.J. Chaplain. Spatio-temporal modelling of the Hes1 and p53-Mdm2 intracellular signalling pathways. Journal of Theoretical Biology, 2011, 273 (1), pp.15. 10.1016/j.jtbi.2010.12.016 . hal-00669200

\section{HAL Id: hal-00669200 https://hal.science/hal-00669200}

Submitted on 12 Feb 2012

HAL is a multi-disciplinary open access archive for the deposit and dissemination of scientific research documents, whether they are published or not. The documents may come from teaching and research institutions in France or abroad, or from public or private research centers.
L'archive ouverte pluridisciplinaire HAL, est destinée au dépôt et à la diffusion de documents scientifiques de niveau recherche, publiés ou non, émanant des établissements d'enseignement et de recherche français ou étrangers, des laboratoires publics ou privés. 


\section{Author's Accepted Manuscript}

Spatio-temporal modelling of the Hes1 and p53Mdm2 intracellular signalling pathways

Marc Sturrock, Alan J. Terry, Dimitris P. Xirodimas, Alastair M. Thompson, Mark A.J. Chaplain

PII: $\quad$ S0022-5193(10)00671-5

DOI: doi:10.1016/j.jtbi.2010.12.016

Reference: YJTBI 6285

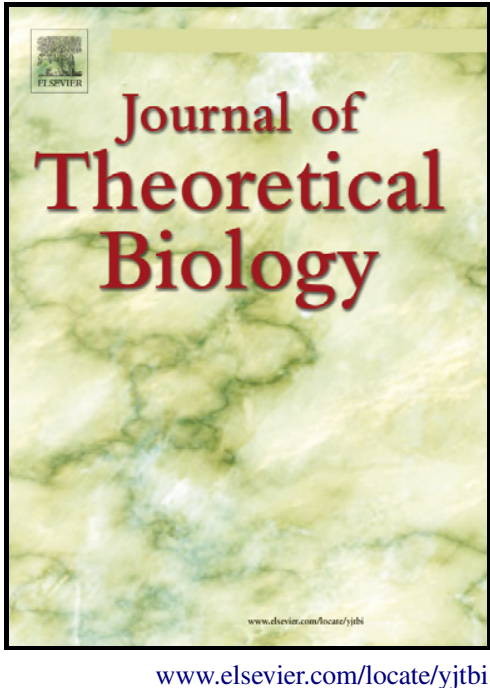

To appear in: $\quad$ Journal of Theoretical Biology

Received date: $\quad 1$ July 2010

Revised date: $\quad 8$ December 2010

Accepted date: 10 December 2010

Cite this article as: Marc Sturrock, Alan J. Terry, Dimitris P. Xirodimas, Alastair M. Thompson and Mark A.J. Chaplain, Spatio-temporal modelling of the Hes1 and p53-Mdm2 intracellular signalling pathways, Journal of Theoretical Biology, doi:10.1016/j.jtbi.2010.12.016

This is a PDF file of an unedited manuscript that has been accepted for publication. As a service to our customers we are providing this early version of the manuscript. The manuscript will undergo copyediting, typesetting, and review of the resulting galley proof before it is published in its final citable form. Please note that during the production process errors may be discovered which could affect the content, and all legal disclaimers that apply to the journal pertain. 


\title{
Spatio-temporal modelling of the Hes1 and p53-Mdm2 intracellular signalling pathways
}

\author{
Marc Sturrock $^{a, *}$, Alan J. Terry ${ }^{a}$, Dimitris P. Xirodimas ${ }^{b}$, \\ Alastair M. Thompson ${ }^{c, d}$, Mark A. J. Chaplain ${ }^{a}$ \\ ${ }^{a}$ Division of Mathematics, University of Dundee, Dundee, DD1 4HN, UK \\ ${ }^{b}$ Wellcome Trust Centre for Gene Regulation \& Expression, College of Life Sciences, University \\ of Dundee, Dundee, DD1 5EH, UK \\ ${ }^{c}$ Department of Surgery \& Molecular Oncology, University of Dundee, Ninewells Hospital and \\ Medical School, Dundee, DD1 9SY, UK \\ ${ }^{d}$ Department of Surgical Oncology, MD Anderson Cancer Center, 1400 Holcombe Boulevard, \\ Houston 77030, USA
}

\begin{abstract}
The correct localisation of transcription factors is vitally important for the proper functioning of many intracellular signalling pathways. Experimental data has shown that many pathways exhibit oscillations in concentrations of the substances involved, both temporally and spatially. Negative feedback loops are important components of these oscillations, providing fine regulation for the factors involved. In this paper we consider mathematical models of two such pathways - Hes1 and p53-Mdm2.

Building on previous mathematical modelling approaches, we derive systems of partial differential equations to capture the evolution in space and time of the variables in the Hes1 and p53-Mdm2 systems. Through computational simulations we show that our reaction-diffusion models are able to produce sustained oscillations both spatially and temporally, accurately reflecting experimental evidence and advancing previous models. The simulations of our models also allow us to calculate a diffusion coefficient range for the variables in each mRNA and protein system, as well as ranges for other key parameters of the models, where sustained oscillations are observed. Finally, by exploiting the explicitly spatial nature of the partial differential equations, we are also able to manipulate mathematically the spatial location of the ribosomes, thus controlling where the proteins are synthesized within the cytoplasm. The results of these simulations predict an optimal distance outside the nucleus where protein synthesis should take place in order to generate sustained oscillations.
\end{abstract}


Using partial differential equation models, new information can be gained about the precise spatio-temporal dynamics of mRNA and proteins. The ability to determine spatial localisation of proteins within the cell is likely to yield fresh insight into a range of cellular diseases such as diabetes and cancer.

Keywords: Intracellular signalling, Negative feedback, Oscillatory dynamics, Spatial model, Hes1, p53, Cancer.

*Corresponding author.

E-mail address: msturrock@maths.dundee.ac.uk

\section{Introduction}

Negative feedback loops controlling the concentrations of key intracellular proteins are prevalent in a diverse range of important cellular processes. Examples include inflammation, meiosis, apoptosis and the heat shock response (Alberts et al. 2008; Lahav et al. 2004; Fall et al. 2002). Experimental data reveal that pathways containing negative feedback loops exhibit sustained oscillations (Hirata et al. 2002; Geva-Zatorsky et al. 2006; Nelson et al. 2004; Shankaran et al. 2009). This is not unexpected given the interactions involved in a negative feedback loop.

[Fig. 1 about here.]

A generic example of a negative feedback loop with variables $\mathrm{x}$ and $\mathrm{y}$ is shown in Fig. 1. An increase in $x$ causes $y$ to increase, which in turn results in the inhibition of $\mathrm{x}$. After $\mathrm{x}$ begins to decrease, this will also cause $\mathrm{y}$ levels to diminish, eventually allowing $\mathrm{x}$ to increase again. This process repeats and thus produces oscillations in $\mathrm{x}$ and $\mathrm{y}$. We will consider intracellular negative feedback loops in this paper, specifically those involving transcription factors, critical contributors to cellular homeostasis and, when dysfunctional, to disease processes. Transcription factors are regulatory proteins required to initiate or regulate transcription in eukaryotic cells. They act by binding to specific DNA sequences in the nucleus, either promoting or inhibiting the binding of RNA polymerase to DNA and are of fundamental importance for normal cellular function. mRNA is transcribed in the nucleus and subsequently protein is translated in the cytoplasm.

Most previous mathematical models examining intracellular negative feedback systems have taken a simplified approach using ordinary differential equations (ODEs) and have not considered the different spatial structures within a cell. 
Such ODE models have used delays to account for the processes of transcription, translation and transport within the cell. However, in this paper we consider modelling the spatial interactions explicitly, using partial differential equation (PDE) models, with the knowledge that the localisation of certain proteins is critical for normal cellular functioning.

The format of this paper is as follows. In the next section, we begin by considering a simple example of a feedback inhibition system, namely that of hes1 mRNA and Hes 1 protein. We present a mathematical model of this system, showing how our model builds on previous work to reflect the biology in greater depth and present our computational simulation results. In the subsequent section we consider the p53-Mdm2 system, where localisation of proteins is of particular importance since it has implications for cancer. Again we develop a mathematical model, justifying the formulation and present our simulation results. In the final section, we conclude with a discussion of our results and an indication of future work in this area.

\section{The Hes1 System}

One of the most thoroughly investigated feedback inhibition systems, and therefore a good place to begin modelling, is the system involving the transcription factor Hes1 (Monk 2003; Momiji and Monk 2008; Agrawal et al. 2009). Hes1 plays a role in somitogenesis, the developmental process whereby the vertebrate embryo becomes segmented. Somitogenesis depends on a segmentation clock. The existence of a direct feedback inhibition loop, coupled with available experimental data (Hirata et al. 2002), suggests that the oscillatory expression of Hes factors play a central role in maintaining the segmentation clock. Hes1 represses the transcription of its own gene through direct binding to regulatory sequences in the hes 1 promoter. The basic interactions of this system (see Fig. 2) are the same as those described previously for the generic variables $\mathrm{x}$ and $\mathrm{y}$. Hes1 protein is produced by hes 1 mRNA and then goes on to inhibit its own mRNA and so forth, with the result that the system oscillates with a period of around 120 minutes.

[Fig. 2 about here.]

\subsection{Hes1 Mathematical Model}

Mathematical modelling of intracellular regulatory systems began with the work of Goodwin (Goodwin 1965). Mahaffy and co-workers developed this work 
introducing spatial structure (although only in 1-dimension) and also delays accounting for transcription and translation (Mahaffy and Pao 1984; Busenberg and Mahaffy 1985; Mahaffy 1988). However, Monk was the first to develop a mathematical model of the Hes1 system and validate it with biological data (Monk 2003). Denoting by [m] and [p] the concentrations of hes 1 mRNA and Hes1 protein respectively, the basic reaction kinetics for this system can be modelled using ordinary differential equations (ODEs) as follows:

$$
\begin{aligned}
& \frac{d[m]}{d t}=\frac{\alpha_{m}}{1+([p] / \hat{p})^{h}}-\mu_{m}[m], \\
& \frac{d[p]}{d t}=\alpha_{p}[m]-\mu_{p}[p] .
\end{aligned}
$$

The first term on the right hand side of Eq. (1) is a Hill function, which decreases as the protein concentration increases, modelling repression by the Hes1 protein. The parameter $\alpha_{m}$ is the basal rate of transcript initiation in the absence of Hes1 protein and $\hat{p}$ is the concentration of Hes 1 that reduces the rate of initiation of hes 1 transcripts to half of its basal value (the repression threshold) and $h$ is a Hill coefficient. The second term represents the natural degradation of the hes1 mRNA, with parameter $\mu_{m}$. The first term on the right hand side of Eq. (2) is the Hes1 protein production term from translation of hes 1 mRNA with parameter $\alpha_{p}$ and the second term represents Hes 1 protein degradation with parameter $\mu_{p}$. It can be proved mathematically that it is impossible for Eqs. (1), (2) to generate sustained oscillatory dynamics (Lewis 2003). In order to model the intracellular processes more accurately, Monk (Monk 2003) introduced delays to Eqs. (1), (2) to account for the processes of transcription and translation. This leads to a system of delay differential equations (DDEs):

$$
\begin{aligned}
\frac{d[m]}{d t} & =\frac{\alpha_{m}}{1+\left(\left[p\left(t-\tau_{m}\right)\right] / \hat{p}\right)^{h}}-\mu_{m}[m], \\
\frac{d[p]}{d t} & =\alpha_{p}\left[m\left(t-\tau_{p}\right)\right]-\mu_{p}[p]
\end{aligned}
$$


where $\tau_{m}$ and $\tau_{p}$ are transcriptional and translational delays, respectively. With the addition of the delay terms, Monk showed that it was possible to obtain sustained oscillations (Monk 2003). These results accurately reflect experimental data well but shed no light on where the mRNA and protein are located in the cell.

We now extend the above models and consider spatial interactions within the cell explicitly as shown schematically in Fig. 3, i.e., we consider the nucleus and cytoplasm as two distinct spatial compartments separated by the nuclear membrane, and the cytoplasm enclosed within the outer cell membrane. Transcription occurs exclusively in the nucleus and protein synthesis occurs exclusively in the cytoplasm. We assume that the main mechanism governing the spatial movement of mRNA and protein between the nucleus and cytoplasm is diffusion. Denoting by $\left[m_{n}\right],\left[m_{c}\right]$ and $\left[p_{n}\right],\left[p_{c}\right]$ the concentrations of nuclear and cytoplasmic hes 1 mRNA and nuclear and cytoplasmic Hes1 protein respectively, the system of equations describing the spatio-temporal evolution of hes1 mRNA and Hes1 protein concentrations is now

$$
\begin{aligned}
& \frac{\partial\left[m_{n}\right]}{\partial t}=D_{m_{n}} \nabla^{2}\left[m_{n}\right]+\underbrace{\frac{\alpha_{m}}{1+\left(\left[p_{n}\right] / \hat{p}\right)^{h}}}_{\text {transcription }}-\mu_{m}\left[m_{n}\right] \\
& \frac{\partial\left[m_{c}\right]}{\partial t}=D_{m_{c}} \nabla^{2}\left[m_{c}\right]-\mu_{m}\left[m_{c}\right], \\
& \frac{\partial\left[p_{c}\right]}{\partial t}=D_{p_{c}} \nabla^{2}\left[p_{c}\right]+\underbrace{\alpha_{p}\left[m_{c}\right]}_{\text {synthesis }}-\mu_{p}\left[p_{c}\right], \\
& \frac{\partial\left[p_{n}\right]}{\partial t}=D_{p_{n}} \nabla^{2}\left[p_{n}\right]-\mu_{p}\left[p_{n}\right],
\end{aligned}
$$

where parameters $D_{i}$ denote the diffusion coefficients for each species. We apply continuity of flux boundary conditions across the (internal) nuclear membrane and zero-flux boundary conditions at the outer cell membrane: 


$$
\begin{aligned}
D_{m_{n}} \frac{\partial\left[m_{n}\right]}{\partial \mathbf{n}} & =D_{m_{c}} \frac{\partial\left[m_{c}\right]}{\partial \mathbf{n}} \text { and }\left[m_{n}\right]=\left[m_{c}\right] \text { at the nuclear membrane, } \\
D_{p_{n}} \frac{\partial\left[p_{n}\right]}{\partial \mathbf{n}} & =D_{p_{c}} \frac{\partial\left[p_{c}\right]}{\partial \mathbf{n}} \text { and }\left[p_{n}\right]=\left[p_{c}\right] \text { at the nuclear membrane, } \\
\frac{\partial\left[m_{c}\right]}{\partial \mathbf{n}} & =0 \quad \text { at the cell membrane, } \\
\frac{\partial\left[p_{c}\right]}{\partial \mathbf{n}} & =0 \quad \text { at the cell membrane, }
\end{aligned}
$$

where $\mathbf{n}$ is a unit normal. In biological terms the boundary conditions allow import and export of mRNA and protein across the nuclear membrane, while ensuring that all molecules remain within the cell and are not exported across the cell membrane. The system is closed by imposing appropriate initial conditions for the mRNA and protein concentrations.

Eqs. (5) - (8) represent a system of reaction-diffusion equations modelling the spatio-temporal evolution of the Hes1 system. The same reaction kinetics from the ODE model (1), (2), are retained but are now also coupled with diffusion to model explicitly protein and mRNA transport within a cell, i.e., molecules move from the nucleus to the cytoplasm and cytoplasm to nucleus across the nuclear membrane. The PDE system reflects the reality mRNA is transcribed from DNA exclusively in the nucleus and that protein is translated from mRNA exclusively in the cytoplasm, i.e., there are production terms only for $\left[m_{n}\right]$ (in equation (5)) and $\left[p_{c}\right]$ (in equation (7)). Finally, we make the assumption that the translation of proteins from mRNA in the cytoplasm occurs some distance away from the nucleus and outside the endoplasmic reticulum (ER), since proteins produced in the ER are mainly either exported to the exterior of the cell or transported to other membrane structures such as the Golgi apparatus, lysosomes and endosomes (see Appendix for details) (Alberts et al. 2008; Mahaffy and Pao 1984; Busenberg and Mahaffy 1985). In order to model this, we modify Eq. (7) as follows:

$$
\frac{\partial\left[p_{c}\right]}{\partial t}=D_{p_{c}} \nabla^{2}\left[p_{c}\right]+H_{1}(x, y) \alpha_{p}\left[m_{c}\right]-\mu_{p}\left[p_{c}\right]
$$

where $H_{1}(x, y)$ is a function localising the protein production whose specific form will be given after the nondimensionalisation of the system.

[Fig. 3 about here.] 
We nondimensionalise (5), (6), (13) and (8) with appropriate reference values (scaling variables) as follows:

$$
\left[\bar{m}_{n}\right]=\frac{\left[m_{n}\right]}{\left[m_{0}\right]},\left[\bar{m}_{c}\right]=\frac{\left[m_{c}\right]}{\left[m_{0}\right]},\left[\bar{p}_{n}\right]=\frac{\left[p_{n}\right]}{\left[p_{0}\right]},\left[\bar{p}_{c}\right]=\frac{\left[p_{c}\right]}{\left[p_{0}\right]}, \bar{t}=\frac{t}{\tau}, \bar{x}=\frac{x}{L}, \bar{y}=\frac{y}{L}
$$

where $\left[m_{0}\right],\left[p_{0}\right]$ are reference concentrations, $\tau$ is a reference time, and $\mathrm{L}$ is a reference length. Using this scaling, (5), (6), (13) and (8) become:

$$
\begin{aligned}
& \frac{\partial\left[\bar{m}_{n}\right]}{\partial \bar{t}}=D_{m_{n}}^{*} \nabla^{2}\left[\bar{m}_{n}\right]+\frac{\alpha_{m}^{*}}{1+\left(p^{*}\left[\bar{p}_{n}\right]\right)^{h}}-\mu_{m}^{*}\left[\bar{m}_{n}\right], \\
& \frac{\partial\left[\bar{m}_{c}\right]}{\partial \bar{t}}=D_{m_{c}}^{*} \nabla^{2}\left[\bar{m}_{c}\right]-\mu_{m}^{*}\left[\bar{m}_{c}\right], \\
& \frac{\partial\left[\bar{p}_{c}\right]}{\partial \bar{t}}=D_{p_{c}}^{*} \nabla^{2}\left[\bar{p}_{c}\right]+H_{1}(\bar{x}, \bar{y}) \alpha_{p}^{*}\left[\bar{m}_{c}\right]-\mu_{p}^{*}\left[\bar{p}_{c}\right], \\
& \frac{\partial\left[\bar{p}_{n}\right]}{\partial \bar{t}}=D_{p_{n}}^{*} \nabla^{2}\left[\bar{p}_{n}\right]-\mu_{p}^{*}\left[\bar{p}_{n}\right],
\end{aligned}
$$

where

$$
\begin{aligned}
& D_{m_{n}}^{*}=\frac{\tau D_{m_{n}}}{L^{2}}, \alpha_{m}^{*}=\frac{\tau \alpha_{m}}{\left[m_{0}\right]}, p^{*}=\frac{\left[p_{0}\right]}{\hat{p}}, \mu_{m}^{*}=\tau \mu_{m}, D_{m_{c}}^{*}=\frac{\tau D_{m_{c}}}{L^{2}} \\
& D_{p_{c}}^{*}=\frac{\tau D_{p_{c}}}{L^{2}}, \alpha_{p}^{*}=\frac{\tau\left[m_{0}\right] \alpha_{p}}{\left[p_{0}\right]}, \mu_{p}^{*}=\tau \mu_{p}, D_{p_{n}}^{*}=\frac{\tau D_{p_{n}}}{L^{2}}
\end{aligned}
$$

and

$$
H_{1}(\bar{x}, \bar{y})= \begin{cases}0, & \text { if } \frac{\bar{x}^{2}}{2}+\bar{y}^{2} \leq 0.25 \\ 1, & \text { if } \frac{\bar{x}^{2}}{2}+\bar{y}^{2}>0.25\end{cases}
$$


$H_{1}(\bar{x}, \bar{y})$ is illustrated graphically in the Appendix. We apply zero initial conditions, zero-flux boundary conditions at the cell membrane and flux continuity boundary conditions across the nuclear membrane:

$$
\begin{aligned}
& {\left[\bar{m}_{n}\right]=\left[\bar{m}_{c}\right]=\left[\bar{p}_{n}\right]=\left[\bar{p}_{c}\right]=0, \text { at } t=0, } \\
& D_{m_{n}}^{*} \frac{\partial\left[\bar{m}_{n}\right]}{\partial \mathbf{n}}=D_{m_{c}}^{*} \frac{\partial\left[\bar{m}_{c}\right]}{\partial \mathbf{n}} \text { and }\left[\bar{m}_{n}\right]=\left[\bar{m}_{c}\right] \text { at nuclear membrane, } \\
& D_{p_{n}}^{*} \frac{\partial\left[\bar{p}_{n}\right]}{\partial \mathbf{n}}=D_{p_{c}}^{*} \frac{\partial\left[\bar{p}_{c}\right]}{\partial \mathbf{n}} \text { and }\left[\bar{p}_{n}\right]=\left[\bar{p}_{c}\right] \text { at nuclear membrane, } \\
& \frac{\partial\left[\bar{m}_{c}\right]}{\partial \mathbf{n}}=0 \text { at cell membrane, } \\
& \frac{\partial\left[\bar{p}_{c}\right]}{\partial \mathbf{n}}=0 \text { at cell membrane. }
\end{aligned}
$$

In the absence of available experimental data (cf. Monk (2003)), we take reference concentrations to be $\left[m_{0}\right]=0.05 \mu \mathrm{M}$ and $\left[p_{0}\right]=1 \mu \mathrm{M}$ (in line with the relative concentrations of mRNA and protein, and also in line with estimates for equivalent p53 data which will be discussed in Section 3.1, Ma et al. (2005)). In preliminary simulations of (15) - (18) it was observed that a period of oscillation was approximately 200 time units. Hence, knowing that the period of oscillation of Hes1 is approximately 2 hours (Hirata et al. 2002), we obtain an appropriate reference time $\tau$ as follows: $200 \tau=2 \mathrm{hrs}$ which implies $\tau=36 \mathrm{~s}$.

For our simulations we use a 2-dimensional cell domain composed of two ellipses to represent the nucleus and cytoplasm. The nucleus has a major axis of length 0.8 units and minor axis of length 0.5 units. The cytoplasm has a major axis of length 3 units and a minor axis of length 2 units. We assume a cell to be of length $30 \mu \mathrm{m}$, and hence take the reference length $\mathrm{L}=10 \mu \mathrm{m}$, i.e., in Figs. 6 and 7 the nondimensional cell width is equal to 3 units or $3 \mathrm{~L}=30 \mu \mathrm{m}$.

\subsubsection{Parameter Estimation}

The following parameter values were used in our simulations of the nondimensional Hes 1 system:

$$
\begin{aligned}
& D_{m_{n}}^{*}=D_{m_{c}}^{*}=D_{p_{c}}^{*}=D_{p_{n}}^{*}=7.5 \times 10^{-4}, \alpha_{m}^{*}=1, \\
& h=5, p^{*}=1, \mu_{m}^{*}=\mu_{p}^{*}=0.03, \alpha_{p}^{*}=2 .
\end{aligned}
$$

From (19) and (25) the dimensional value of the diffusion coefficient, $D_{m_{n}}$, can be calculated:

$$
D_{m_{n}}=\frac{L^{2} D_{m_{n}}^{*}}{\tau}=2.08 \times 10^{-11} \mathrm{~cm}^{2} \mathrm{~s}^{-1} .
$$


Similarly, we are able to calculate the remaining dimensional parameter values:

$$
\begin{aligned}
& D_{m_{n}}=D_{m_{c}}=D_{p_{c}}=D_{p_{n}}=2.08 \times 10^{-11} \mathrm{~cm}^{2} \mathrm{~s}^{-1}, \alpha_{m}=0.02 \mathrm{Ms}^{-1}, \\
& h=5, \hat{p}=1 \times 10^{-6} \mathrm{M}, \mu_{m}=\mu_{p}=8.33 \times 10^{-4} \mathrm{~s}^{-1}, \alpha_{p}=1.11 \mathrm{~s}^{-1} .
\end{aligned}
$$

From a number of simulations carried out on system (15) - (18), we have calculated a range of diffusion coefficients (if all other parameters remain unchanged) for mRNA and protein for which the system exhibits oscillatory dynamics: $1.67 \times 10^{-11}$ to $9.72 \times 10^{-11} \mathrm{~cm}^{2} \mathrm{~s}^{-1}$. We have also calculated a range of mRNA degradation rates $\left(1.67 \times 10^{-4}\right.$ to $\left.1.17 \times 10^{-3} \mathrm{~s}^{-1}\right)$, protein degradation rates $\left(1.94 \times 10^{-4}\right.$ to $\left.1.06 \times 10^{-3} \mathrm{~s}^{-1}\right)$ and Hill coefficients $(\mathrm{h} \geq 4)$, for which the system exhibits oscillations.

We note that the kinetic parameters we have used are in line with previous estimates (Monk 2003). Regarding the values of our diffusion coefficients, we note that the results of Klonis et al. (2002) show that diffusion rates of macromolecules in the cytoplasm and nucleus are up to 100-times slower than in aqueous buffers, which would then bring our estimated values of around $10^{-11}-10^{-10} \mathrm{~cm}^{2} \mathrm{~s}^{-1}$ in line with the estimates of Seksek et al. (1997) $\left(10^{-8} \mathrm{~cm}^{2} \mathrm{~s}^{-1}\right)$ and Matsuda et al. (2008) $\left(10^{-9} \mathrm{~cm}^{2} \mathrm{~s}^{-1}\right)$.

\subsection{Hes1 Numerical Simulations}

We solved PDE system (15) - (18) numerically using the COMSOL/FEMLAB package, which uses the finite element technique. Triangular basis elements and Lagrange quadratic basis functions along with a backward Euler time-stepping method for integrating the equations were used in all simulations.

Fig. 4 shows the total concentrations of hes $1 \mathrm{mRNA}$ and Hes1 protein over time in the nuclear compartment, while Fig. 5 shows the total concentrations in the cytoplasm. Both sets of results show oscillatory dynamics of the Hes1 system.

[Fig. 4 about here.]

[Fig. 5 about here.]

[Fig. 6 about here.]

The plots presented in Figs. 6 and 7 show how the hes 1 mRNA and protein concentrations vary spatially as well as temporally within the cell. The mRNA is produced inside the nucleus and by $t=60$ minutes has started to cross the nuclear membrane to enter the cytoplasm (Fig. 6). In the cytoplasm the mRNA 
is translated into protein, which then diffuses back into the nucleus and represses the production of its own mRNA ( $\mathrm{t}=120$ minutes). The mRNA concentration has clearly depleted by $\mathrm{t}=120$ minutes, reflecting the period of the temporal oscillation seen in Figs. 4, 5. As can be seen from Fig. 7, there is a delay in the rise of protein concentration after $\mathrm{t}=0$ as it takes time for the mRNA to be produced and exported to the cytoplasm. By $\mathrm{t}=60$ minutes the protein levels have clearly risen in the cytoplasm and have reached the nucleus. At $t=120$ minutes the protein concentration has decreased significantly, due to the inhibition of mRNA transcription by the protein.

[Fig. 7 about here.]

Furthermore we have performed simulations of the Hes1 system on 3D spherical and ellipsoidal domains. In Fig. 8 we present snapshots of the Hes1 protein concentration in the cytoplasm obtained from 3-dimensional simulations of system (15) - (18) on both spherical and ellipsoidal domains (all parameter values as per previous simulations). We found that using different domain geometries (of approximately the same volume) does not affect the solution qualitatively, i.e., oscillatory solutions were still observed. However, as is to be expected, the precise spatial distribution of concentrations at a given time varies between the different geometries.

[Fig. 8 about here.]

The simulation results from our system (15) - (18) reflect qualitatively the dynamics of the Hes 1 pathway, a simple example of control of protein expression by a negative feedback loop (Hirata et al. 2002).

The main advantage of using a system of PDEs to model intracellular reactions is that the PDEs enable spatial effects to be examined explicitly. To this end, we carried out a number of simulations on system (15) - (18), where we varied the values of the diffusion coefficients (all other parameters remaining unchanged) of the mRNA and protein. By doing this we found a range of values for the diffusion coefficients $D_{m_{n}}, D_{m_{c}}, D_{p_{c}}, D_{p_{n}}$ where the system exhibits oscillatory dynamics, i.e., for $1.67 \times 10^{-11} \mathrm{~cm}^{2} \mathrm{~s}^{-1} \leq D_{m}, D_{p} \leq 9.72 \times 10^{-11} \mathrm{~cm}^{2} \mathrm{~s}^{-1}$ oscillations were observed, while no oscillations were observed outside this range. Hence if diffusion of mRNA and protein is either too slow or too fast, oscillations do not occur. In a similar manner, we also calculated a range of mRNA degradation rates $\left(\mu_{m}\right.$ from $1.67 \times 10^{-4}$ to $\left.1.17 \times 10^{-3} \mathrm{~s}^{-1}\right)$, protein degradation rates $\left(\mu_{p}\right.$ 
from $1.94 \times 10^{-4}$ to $\left.1.06 \times 10^{-3} \mathrm{~s}^{-1}\right)$ and Hill coefficients $(\mathrm{h} \geq 4)$, for which the system exhibits oscillations.

In order to examine further the influence of spatial effects on the oscillatory dynamics, in the next section we investigate the role of the function $H_{1}(\bar{x}, \bar{y})$ which governs the production of the Hes 1 protein in the cytoplasm (see equation (17)).

\subsection{Varying the Location of Protein Synthesis in the Hes1 System}

In this section we present a number of numerical simulations of the PDE system (15) - (18) demonstrating the effect of varying the function, $H_{1}(\bar{x}, \bar{y})$, defined in section 2.1 and shown graphically in the Appendix. This function controls the spatial location of Hes 1 protein production in the cytoplasm which must take place away from the endoplasmic reticulum.

As can be seen from the simulation results shown in Figs. 9, 10 (top row), if protein production is located too close to the nucleus, the amplitude of the oscillations is much reduced and is not in line with experimental observations. In a similar manner, the plots in the bottom row of Figs. 9, 10 show that when protein synthesis is located too far from the nucleus, the oscillations are much reduced in amplitude or disappear altogether (with the concentrations tending to a steady state). The plots in the middle row demonstrate sustained oscillations in the mRNA and protein levels (in both the nucleus and cytoplasm) when protein synthesis is located at a "moderate" distance from the nucleus. The simulations therefore reveal an "optimum" distance outside the nucleus for protein production for which undamped oscillations of large amplitude are observed. Snapshots of the corresponding spatial plots of the Hes 1 protein concentration are presented in Fig. 11.

[Fig. 9 about here.]

[Fig. 10 about here.]

[Fig. 11 about here.]

\subsection{Inhibition of the Proteasome}

Our previous simulation results have shown that oscillatory dynamics in the Hes 1 system occur only for a suitable protein degradation rate $\mu_{p}$. Experiments have demonstrated that in the presence of the proteasome inhibitor MG132, hes1 mRNA is transiently induced by a serum treatment, but is then suppressed persistently thereafter (Hirata et al. 2002). We now show the result of inhibiting the 
proteasome in the Hes1 model (15) - (18) by reducing the decay rate for Hes1 protein $\mu_{p}$ by a factor of 10 .

The following parameter set is therefore used to simulate the effect of suppressing the proteasome:

$$
\begin{aligned}
& D_{m_{n}}^{*}=D_{m_{c}}^{*}=D_{p_{c}}^{*}=D_{p_{n}}^{*}=7.5 \times 10^{-4}, \alpha_{m}^{*}=1, \\
& h=5, p^{*}=1, \mu_{m}^{*}=0.03, \mu_{p}^{*}=0.003, \alpha_{p}^{*}=2 .
\end{aligned}
$$

Fig. 12 shows a plot of the total concentrations of hes 1 mRNA (red) and Hes 1 protein (blue) in the nucleus over time, while Fig. 13 shows the corresponding concentrations in the cytoplasm. Finally, Figs. 14 and 15 show the spatio-temporal evolution of the mRNA and protein concentrations respectively over the same time period. As can be seen from all these plots, no oscillations in the concentration levels are observed, in line with the experimental results of (Hirata et al. 2002).

Having developed the PDE model (15) - (18) for the Hes1 system and explored its spatio-temporal dynamics through a range of simulations, in the next section we turn our attention to the $\mathrm{p} 53-\mathrm{Mdm} 2$ system where a negative feedback loop also exists.

[Fig. 12 about here.]

[Fig. 13 about here.]

[Fig. 14 about here.]

[Fig. 15 about here.]

\section{The p53-Mdm2 System}

The pleiotropic p53 tumour suppressor protein is a well-established regulator of the cell cycle. In response to a variety of cellular stresses, such as DNA damage, ribosome biogenesis defects, oncogene activation, hypoxia and chemotherapeutic drugs, p53 is activated and induces a range of responses including cell cycle arrest, senescence or apoptosis (programmed cell death) (Vousden and Prives 2009; Vogelstein et al. 2000). The central role for $\mathrm{p} 53$ as a cell cycle regulator is highlighted in human cancers. Mutations that inactivate p53 function have been detected in more than $50 \%$ of human cancers (Bennet et al. 1999). Importantly, even tumours with wild type p53 have defects in upstream regulators or downstream effectors 
of $\mathrm{p} 53$. Therefore, inactivation of the p53 pathway is a common event in cancer development (Zilfou and Lowe 2009; Toledo and Wahl 2006).

In normal unstressed conditions, the levels and activity of p53 remain low, but in response to cellular stress, p53 levels are increased and the p53 pathway is activated. A vital negative regulator of p53 function in cells is the Mdm2 oncogene product. Mdm2 suppresses p53 function by at least two mechanisms. Firstly, Mdm2 interacts with the transactivation domain of p53 in the N-terminus inhibiting p53 transcriptional activity and also acts as a ubiquitin E3-ligase such that $\mathrm{Mdm} 2$ promotes $\mathrm{p} 53$ ubiquitination and proteasomal degradation. Secondly, $\mathrm{Mdm} 2$ is a target gene for $\mathrm{p} 53$. This creates a negative feedback loop which provides tight regulation of p53 function in cells (Coutts et al. 2009; Carter and Vousden 2009).

The importance of the p53-Mdm2 feedback loop was demonstrated in mouse animal model systems where deletion of Mdm2 caused embryonic lethality that was rescued by concomitant p53 deletion (Jones et al. 1995; de Oca Luna et al. 1995). Mdm2 is overexpressed in tumours with wild type p53 function, which could account for suppression of p53 function (Toledo and Wahl 2006; Marine and Jochemsen 2003). It has also been observed that Mdm2 protein levels dramatically decrease within the first 5 minutes after DNA damage, which allows for the accumulation of p53 (Stommel and Wahl 2004). Therefore, a key activity of $\mathrm{Mdm} 2$ in cells is to suppress p53 function. Given the importance of p53 in controlling cell cycle and tumour development, it is not surprising that the p53Mdm2 feedback loop is very tightly controlled in cells. Experiments have been performed to measure the dynamics of fluorescently tagged p53 and Mdm2 over several days in individual living cells (Geva-Zatorsky et al. 2006). Some cells exhibited undamped oscillations for at least 3 days (more than 10 peaks).

Mathematical models of the p53-Mdm2 system have taken a variety of forms. One of the earliest models was that of Bar-Or (Bar-Or et al. 2000), which included an unknown intermediary component to the system representing the delayed synthesis of Mdm2 by p53 (despite extensive research into p53-Mdm2 interactions, no such intermediary has been identified to date). It was believed that $\mathrm{p} 53$ and Mdm2 concentrations exhibited damped oscillations in response to DNA damage. However, later experimental results in individual cells showed instead that p53 responded in digital pulses to DNA damage (Geva-Zatorsky et al. 2006; Lahav et al. 2004). As mathematical models have evolved to capture the undamped oscillations revealed by experiments, many different approaches have been adopted, including combining positive feedback loops with negative feedback loops in ODE metapopulation-like models (Ciliberto et al. 2005; Zhang et al. 2007). These mod- 
els were the first to make the important distinction between nuclear and cytoplasmic concentrations. Some models have taken stochastic effects into account (Puszyński et al. 2008; Proctor and Gray 2008; Ouattara et al. 2010) while others have used time delays (Mihalas et al. 2006; Ma et al. 2005; Batchelor et al. 2008), in a manner similar to that discussed for the Hes 1 system previously. An attempt was made to model the spatial aspect of the system by Gordon (Gordon et al. 2009), but this model also relied on time delays to produce oscillations. In the model we present here, we show that by simply considering the spatial interactions explicitly, we can obtain the experimentally observed oscillatory dynamics.

[Fig. 16 about here.]

As a first approximation of the p53-Mdm2 pathway following (Monk 2003), we look at the main interactions (Fig. 16), setting aside post-translational modifications or other proteins that may have an effect on the system. We assume continuous stress on the system (reflecting some physiological stresses such as hypoxia) for simplicity, and as a result of this do not include a DNA damage term. p53 protein is assumed to be made at a constant rate (Monk 2003) in an annular region in the cytoplasm (see Appendix for details). p53 translocates to the nucleus where it acts as a transcription factor, upregulating production of Mdm 2 mRNA. Newly synthesized Mdm2 mRNA is subsequently exported to the cytoplasm where it is translated into protein. Mdm2 protein enhances p53 degradation in both the nucleus and cytoplasm via ubiquitination (Xirodimas et al. 2001). In summary, p53 upregulates Mdm2, but Mdm2 downregulates p53 and so a negative feedback loop exists.

\section{1. p53-Mdm2 Mathematical Model}

We begin by looking at the fundamental reaction kinetics of the system. Denoting the concentrations of p53, Mdm2 and Mdm2 mRNA by [p53], [Mdm2] and $[\mathrm{Mdm} 2 \mathrm{~m}]$ respectively, the ODE system below is formulated to capture the interactions depicted in Fig. 16: 


$$
\begin{aligned}
\frac{d[p 53]}{d t} & =\beta-\left(\mu+v\left(\frac{[M d m 2]^{h_{1}}}{\widehat{M d m 2}^{h_{1}}+[M d m 2]^{h_{1}}}\right)\right)[p 53], \\
\frac{d[M d m 2 m]}{d t} & =\alpha+\eta\left(\frac{[p 53]^{h_{2}}}{\widehat{p 53}^{h_{2}}+[p 53]^{h_{2}}}\right)-\phi[M d m 2 m], \\
\frac{d[M d m 2]}{d t} & =\gamma[M d m 2 m]-\rho[M d m 2] .
\end{aligned}
$$

The ODE describing p53 is composed of a production term $\beta$, followed by a natural degradation term of rate $\mu$, and finally a degradation term dependent on the amount of Mdm2, with parameter $v$. The second ODE, modelling Mdm2 mRNA, has a production term with basal rate $\alpha$, followed by an enhanced production term dependent on the amount of p53, reflecting the activity of p53 as a transcription factor (with maximal initiation rate $\eta$ ), and finally a natural degradation term of rate $\phi$. The final ODE is for Mdm2 protein, which simply has a production term dependent on the amount of Mdm2 mRNA, rate $\gamma$, and a natural degradation term, rate $\rho . \widehat{M d m 2}$ and $\widehat{p 53}$ are activation thresholds, and $h_{1}$ and $h_{2}$ are Hill coefficients. As before with the Hes 1 system, Monk (Monk 2003) added a delay to account for transcript elongation, splicing, processing and export:

$$
\begin{aligned}
\frac{d[p 53]}{d t} & =\beta-\left(\mu+v\left(\frac{[M d m 2]^{h_{1}}}{\widehat{M d m 2}^{h_{1}}+[M d m 2]^{h_{1}}}\right)\right)[p 53], \\
\frac{d[M d m 2 m]}{d t} & =\alpha+\eta\left(\frac{[p 53(t-\tau)]^{h_{2}}}{\widehat{p 53}^{h_{2}}+[p 53(t-\tau)]^{h_{2}}}\right)-\phi[M d m 2 m], \\
\frac{d[M d m 2]}{d t} & =\gamma[M d m 2 m]-\rho[M d m 2] .
\end{aligned}
$$


System (30), (31), (32) produces oscillations (Monk 2003), but does not distinguish between events taking place in the nucleus and cytoplasm. Therefore, we now consider the spatial interactions explicitly, allowing for diffusion within the cell, and arrive at the following system of PDEs:

$$
\begin{aligned}
\frac{\partial\left[p 53_{c}\right]}{\partial t} & =D_{c_{1}} \nabla^{2}\left[p 53_{c}\right]+H_{2}(x, y) \beta-\left(\mu+v\left(\frac{\left[M d m 2_{c}\right]^{h_{1}}}{\widehat{M d m 2}^{h_{1}}+\left[M d m 2_{c}\right]^{h_{1}}}\right)\right)\left[p 53_{c}\right] \\
\frac{\partial\left[p 53_{n}\right]}{\partial t} & =D_{n_{1}} \nabla^{2}\left[p 53_{n}\right]-\left(\mu+v\left(\frac{\left[M d m 2_{n}\right]^{h_{1}}}{\widehat{M d m 2}^{h_{1}}+\left[M d m 2_{n}\right]^{h_{1}}}\right)\right)\left[p 53_{n}\right] \\
\frac{\partial\left[M d m 2 m_{n}\right]}{\partial t} & =D_{n_{2}} \nabla^{2}\left[M d m 2_{n}\right]+\alpha+\eta\left(\frac{\left[p 53_{n}\right]^{h_{2}}}{\widehat{p 53}^{h_{2}}+\left[p 53_{n}\right]^{h_{2}}}\right)-\phi\left[M d m 2 m_{n}\right] \\
\frac{\partial\left[M d m 2 m_{c}\right]}{\partial t} & =D_{c_{2}} \nabla^{2}\left[M d m 2_{c} m_{c}\right]-\phi\left[M d m 2 m_{c}\right], \\
\frac{\partial\left[M d m 2_{c}\right]}{\partial t} & =D_{c_{3}} \nabla^{2}\left[M d m 2_{c}\right]+H_{1}(x, y) \gamma\left[M d m m_{c}\right]-\rho\left[M d m 2_{c}\right]
\end{aligned}
$$

where a subscript " $c$ " denotes a cytoplasmic variable and a subscript " $n$ " denotes a nuclear variable. The parameters $D_{i}$ are the diffusion coefficients for each species and $H_{1}(x, y)$ and $H_{2}(x, y)$ are functions localising the protein production whose specific form will be given after the nondimensionalisation of the system. We apply continuity of flux boundary conditions across the (internal) nuclear membrane 
and zero-flux boundary conditions at the outer cell membrane:

$$
\begin{aligned}
D_{n_{1}} \frac{\partial\left[p 53_{n}\right]}{\partial \mathbf{n}} & =D_{c_{1}} \frac{\partial\left[p 53_{c}\right]}{\partial \mathbf{n}} \text { and }\left[p 53_{n}\right]=\left[p 53_{c}\right] \text { at nuclear membrane, } \\
D_{n_{2}} \frac{\partial\left[M d m 2 m_{n}\right]}{\partial \mathbf{n}} & =D_{c_{2}} \frac{\partial\left[M d m 2_{c}\right]}{\partial \mathbf{n}} \text { and }\left[M d m 2 m_{n}\right]=\left[M d m 2 m_{c}\right] \text { at nuclear membrane, } \\
D_{n_{3}} \frac{\partial\left[M d m 2_{n}\right]}{\partial \mathbf{n}} & =D_{c_{3}} \frac{\partial\left[M d m 2_{c}\right]}{\partial \mathbf{n}} \text { and }\left[M d m 2_{n}\right]=\left[M d m 2_{c}\right] \text { at nuclear membrane, } \\
\frac{\partial\left[p 53_{c}\right]}{\partial \mathbf{n}} & =0 \text { at cell membrane, } \\
\frac{\partial\left[M d m 2 m_{c}\right]}{\partial \mathbf{n}} & =0 \text { at cell membrane, } \\
\frac{\partial\left[M d m 2_{c}\right]}{\partial \mathbf{n}} & =0 \text { at cell membrane, }
\end{aligned}
$$

where $\mathbf{n}$ is a unit normal.

We nondimensionalise (33) - (38) with appropriate reference values as follows:

$$
\begin{aligned}
& {\left[{\overline{p 53_{n}}}_{n}=\frac{\left[p 53_{n}\right]}{\left[p 53_{0}\right]},\left[\overline{p 53_{c}}\right]=\frac{\left[p 53_{c}\right]}{\left[p 53_{0}\right]},\left[\overline{M d m 2 m_{n}}\right]=\frac{\left[M d m 2 m_{n}\right]}{\left[M d m 2 m_{0}\right]}\right.} \\
& {\left[{\overline{M d m 2 m_{c}}}\right]=\frac{\left[M d m 2_{c}\right]}{\left[M d m 2_{m_{0}}\right]},\left[\overline{M d m 2_{n}}\right]=\frac{\left[M d m 2_{n}\right]}{\left[M d m 2_{0}\right]}} \\
& {\left[\overline{M d m 2_{c}}\right]=\frac{\left[M d m 2_{c}\right]}{\left[M d m 2_{0}\right]}, \bar{t}=\frac{t}{\tau}, \bar{x}=\frac{x}{L}, \bar{y}=\frac{y}{L}}
\end{aligned}
$$

where $\left[p 53_{0}\right],\left[M d m 2 m_{0}\right],\left[M d m 2_{0}\right]$ are reference concentrations, $\tau$ is a reference time, and $\mathrm{L}$ is a reference length $(10 \mu \mathrm{m}$ as with Hes 1 system $)$. Using this scaling, (33) - (38) becomes: 


$$
\begin{aligned}
& \begin{array}{l}
\frac{\partial\left[\overline{p 53}_{c}\right]}{\partial \bar{t}}=D_{c_{1}}^{*} \nabla^{2}\left[\overline{p 53}_{c}\right]+H_{2}(\bar{x}, \bar{y}) \beta^{*}-\left(\mu^{*}+v^{*}\left(\frac{\left[\overline{M d m 2}_{c}\right]^{h_{1}}}{M d m 2^{*}+\left[\overline{M d m 2}_{c}\right]^{h_{1}}}\right)\right. \\
\frac{\partial\left[\overline{p 53}_{n}\right]}{\partial \bar{t}}=D_{n_{1}}^{*} \nabla^{2}\left[\overline{p 53}_{n}\right]-\left(\mu^{*}+v^{*}\left(\frac{\left[\overline{M d m 2}_{n}\right]^{h_{1}}}{M d m 2^{*}+\left[\overline{M d m 2}_{n}\right]^{h_{1}}}\right)\right)\left[\overline{p 53}_{n}\right],
\end{array} \\
& \frac{\partial\left[{\overline{M d m 2 m_{n}}}\right]}{\partial \bar{t}}=D_{n_{2}}^{*} \nabla^{2}\left[{\overline{M d m 2 m_{n}}}_{n}\right]+\alpha^{*}+\eta^{*}\left(\frac{\left[\overline{p 53}_{n}\right]^{h_{2}}}{p 53^{*}+\left[\overline{p 53}_{n}\right]^{h_{2}}}\right)-\phi^{*}\left[{\overline{M d m 2 m_{n}}}_{n}\right] \\
& \frac{\partial\left[{\overline{M d m 2 m_{c}}}\right]}{\partial \bar{t}}=D_{c_{2}}^{*} \nabla^{2}\left[\overline{\operatorname{Mdm2}}_{c}\right]-\phi^{*}\left[\overline{\operatorname{Mdm2}}_{c}\right] \\
& \frac{\partial\left[\overline{M d m 2}_{c}\right]}{\partial \bar{t}}=D_{c_{3}}^{*} \nabla^{2}\left[\overline{M d m 2}_{c}\right]+H_{1}(\bar{x}, \bar{y}) \gamma^{*}\left[\overline{M d m 2}_{c}\right]-\rho^{*}\left[\overline{M d m 2}_{c}\right] \\
& \frac{\partial\left[\overline{M d m}_{n}\right]}{\partial \bar{t}}=D_{n_{3}}^{*} \nabla^{2}\left[\overline{\operatorname{Mdm} 2}_{n}\right]-\rho^{*}\left[\overline{\operatorname{Mdm} 2}_{n}\right]
\end{aligned}
$$

where

$$
\begin{aligned}
& D_{c_{1}}^{*}=\frac{\tau D_{c_{1}}}{L^{2}}, \beta^{*}=\frac{\tau \beta}{\left[p 53_{0}\right]}, \mu^{*}=\tau \mu, v^{*}=\tau \nu, M d m 2^{*}=\frac{\widehat{M d m 2}{ }^{h_{1}}}{\left[M d m 2_{0}\right]^{h_{1}}}, \\
& D_{n_{1}}^{*}=\frac{\tau D_{n_{1}}}{L^{2}}, D_{n_{2}}^{*}=\frac{\tau D_{n_{2}}}{L^{2}}, \alpha^{*}=\frac{\tau \alpha}{\left[M d m 2 m_{0}\right]}, \eta^{*}=\frac{\tau \eta}{\left[M d m 2 m_{0}\right]}, \\
& p 53^{*}=\frac{\widehat{p 53} h_{2}}{\left[p 53_{0}\right]^{h_{2}}}, \phi^{*}=\tau \phi, D_{c_{2}}^{*}=\frac{\tau D_{c_{2}}}{L^{2}}, D_{c_{3}}^{*}=\frac{\tau D_{c_{3}}}{L^{2}}, \\
& \gamma^{*}=\frac{\tau \gamma\left[M d m 2 m_{0}\right]}{\left[M d m 2_{0}\right]}, \rho^{*}=\tau \rho, D_{n_{3}}^{*}=\frac{\tau D_{n_{3}}}{L^{2}},
\end{aligned}
$$


and

$$
H_{1}(\bar{x}, \bar{y})=\left\{\begin{array}{cc}
0, & \text { if } \frac{\bar{x}^{2}}{2}+\bar{y}^{2} \leq 0.25 \\
1, & \text { if } \frac{\bar{x}^{2}}{2}+\bar{y}^{2}>0.25
\end{array}\right.
$$

and

$$
H_{2}(\bar{x}, \bar{y})= \begin{cases}0, & \text { if } \frac{\bar{x}^{2}}{2}+\bar{y}^{2} \leq 0.25 \\ 1, & \text { if } 0.25<\frac{\bar{x}^{2}}{2}+\bar{y}^{2}<0.375 \\ 0, & \text { if } \frac{\bar{x}^{2}}{2}+\bar{y}^{2} \geq 0.375 .\end{cases}
$$

$H_{1}(\bar{x}, \bar{y})$ and $H_{2}(\bar{x}, \bar{y})$ are illustrated graphically in the Appendix. We apply zero initial conditions, zero-flux boundary conditions at the cell membrane and flux continuity boundary conditions across the nuclear membrane:

$$
\begin{aligned}
& {\left[\overline{p 53}_{n}\right]=\left[\overline{p 53}_{c}\right]=\left[\overline{M d m 2 m}_{n}\right]=\left[{\overline{M d m 2 m_{c}}}\right]=\left[\overline{M d m 2}_{n}\right]=\left[\overline{M d m 2}_{c}\right]=0, \text { at } t=0 \text {, }} \\
& D_{n_{1}}^{*} \frac{\partial\left[\overline{p 53}_{n}\right]}{\partial \mathbf{n}}=D_{c_{1}}^{*} \frac{\partial\left[\overline{p 53}_{c}\right]}{\partial \mathbf{n}} \text { and }\left[\overline{p 53}_{n}\right]=\left[\overline{p 53}_{c}\right] \text { at nuclear membrane, } \\
& D_{n_{2}}^{*} \frac{\partial\left[{\overline{M d m 2 m_{n}}}_{n}\right.}{\partial \mathbf{n}}=D_{c_{2}}^{*} \frac{\partial\left[{\overline{M d m 2 m_{c}}}\right]}{\partial \mathbf{n}} \text { and }\left[{\overline{M d m 2 m_{n}}}_{n}=\left[{\overline{M d m 2 m_{c}}}_{c}\right]\right. \text { at nuclear membrane, } \\
& D_{n_{3}}^{*} \frac{\partial\left[\overline{M d m}_{n}\right]}{\partial \mathbf{n}}=D_{c_{3}}^{*} \frac{\partial\left[\overline{M d m}_{c}\right]}{\partial \mathbf{n}} \text { and }\left[\overline{M d m 2}_{n}\right]=\left[\overline{M d m 2}_{c}\right] \text { at nuclear membrane, } \\
& \frac{\partial\left[\overline{p 53}_{c}\right]}{\partial \mathbf{n}}=0 \text { at cell membrane, } \\
& \frac{\partial\left[\overline{M d m 2 m}_{c}\right]}{\partial \mathbf{n}}=0 \text { at cell membrane, } \\
& \frac{\partial\left[\overline{M d m 2}_{c}\right]}{\partial \mathbf{n}}=0 \text { at cell membrane, }
\end{aligned}
$$

We found a reference concentration for $\left[p 53_{0}\right]$ of $0.5 \mu \mathrm{M}$, and estimated reference concentrations for $\left[M d m 2 m_{0}\right]$ and $\left[M d m 2_{0}\right]$ to be $0.05 \mu \mathrm{M}$ and $2 \mu \mathrm{M}$ respectively (Ma et al. 2005). In preliminary simulations of (46) - (51) it was observed that a period of oscillation was approximately 400 time units. Knowing the period of oscillation of p53 roughly equals 3 hours (Monk 2003), we can find an appropriate reference time: $400 \tau=3 \mathrm{hrs}$ which implies that $\tau=27 \mathrm{~s}$. 


\subsubsection{Parameter Estimation}

For simulations of the nondimensional p53-Mdm2 system, the following parameter values were used:

$$
\begin{aligned}
& D_{c_{1}}^{*}=D_{n_{1}}^{*}=D_{n_{2}}^{*}=D_{c_{2}}^{*}=D_{c_{3}}^{*}=D_{n_{3}}^{*}=9 \times 10^{-4}, \beta^{*}=0.5, \\
& \mu^{*}=0.003, v^{*}=1, h_{1}=2, M d m 2^{*}=16, \alpha^{*}=0.0175, \eta^{*}=1, \\
& h_{2}=4, p 53^{*}=5, \phi^{*}=0.0175, \gamma^{*}=0.5, \rho^{*}=0.025 .
\end{aligned}
$$

We calculate estimates for parameter values of the dimensional p53-Mdm2 model (33) - (38) using (52) and (60):

$$
\begin{aligned}
& D_{c_{1}}=D_{n_{1}}=D_{n_{2}}=D_{c_{2}}=D_{c_{3}}=D_{n_{3}}=3.33 \times 10^{-11} \mathrm{~cm}^{2} \mathrm{~s}^{-1}, \beta=9.26 \times 10^{-3} \mathrm{Ms}^{-1}, \\
& \mu=1.11 \times 10^{-4} \mathrm{~s}^{-1}, v=0.04 \mathrm{~s}^{-1}, h_{1}=2, \widehat{M d m 2}=8 \times 10^{-6} \mathrm{M}, \\
& \alpha=3.24 \times 10^{-11} \mathrm{Ms}^{-1}, \eta=1.85 \times 10^{-9} \mathrm{Ms}^{-1}, h_{2}=4, \widehat{p 53}=7.48 \times 10^{-7} \mathrm{M}, \\
& \phi=6.48 \times 10^{-4} \mathrm{~s}^{-1}, \gamma=0.74 \mathrm{~s}^{-1}, \rho=9.26 \times 10^{-4} \mathrm{~s}^{-1} .
\end{aligned}
$$

Leaving all other parameter values unchanged, we have calculated a range of diffusion coefficients for all the variables, for which we have sustained oscillations: $7.41 \times 10^{-12}$ to $2.48 \times 10^{-10} \mathrm{~cm}^{2} \mathrm{~s}^{-1}$. In addition to this we calculated the following ranges of degradation rates and Hill coefficients for which, if all other parameters remain unaltered, oscillations are observed: $\mu=0$ to $5.19 \times 10^{-4} \mathrm{~s}^{-1}$, $v=2.67 \times 10^{-3}$ to $1.56 \mathrm{~s}^{-1}, \rho=2.96 \times 10^{-3}$ to $4.30 \times 10^{-3} \mathrm{~s}^{-1}, \phi=2.59 \times 10^{-4}$ to $1.59 \times 10^{-3} \mathrm{~s}^{-1}, h_{1} \geq 2, h_{2} \geq 2$.

Once again, we note that the kinetic parameters we have used are in line with previous estimates (Proctor and Gray 2008). Regarding the values of our diffusion coefficients, we note that the results of Klonis et al. (2002) show that diffusion rates of macromolecules in the cytoplasm and nucleus are up to 100-times

slower than in aqueous buffers, which would then bring our estimated values of around $10^{-12}-10^{-10} \mathrm{~cm}^{2} \mathrm{~s}^{-1}$ in line with the estimates of Seksek et al. (1997) $\left(10^{-8} \mathrm{~cm}^{2} \mathrm{~s}^{-1}\right)$ and Matsuda et al. (2008) $\left(10^{-9} \mathrm{~cm}^{2} \mathrm{~s}^{-1}\right)$.

\section{2. p53-Mdm2 Numerical Simulations}

As in the case of the Hes1 system, we solved PDE system (46) - (51) numerically using the COMSOL/FEMLAB package. For all our simulations we used the same 2-dimensional cell domain composed of two ellipses to represent 
the nucleus and cytoplasm. Figs. 17 and 18 show the concentrations of p53 and $\mathrm{Mdm} 2$ in the nucleus and cytoplasm obtained from computational simulations of the $\mathrm{p} 53-\mathrm{Mdm} 2$ system.

[Fig. 17 about here.]

[Fig. 18 about here.]

As with the Hes1 system simulations, we can see from these p53 simulations that the mRNA concentration is higher in the nucleus compared to the protein concentrations and vice versa for the cytoplasmic compartment. The relative ratio of nuclear and cytoplasmic concentrations of a protein can be easily obtained from our model but is difficult to measure experimentally. We could not find any existing experimental evidence in the literature to confirm this observation and therefore we leave it as a prediction of the model.

Figs. 19 and 20 show how the dynamics of the p53-Mdm2 system evolve in space as well as time. From Fig. 19, we see that p53 has accumulated in the cytoplasm at $\mathrm{t}=45$ minutes. The $\mathrm{p} 53$ then begins to diffuse across the nuclear boundary and enters the nucleus $(\mathrm{t}=90$ minutes). The presence of p53 in the nucleus upregulates the expression of Mdm2 which results in enhanced decay of $\mathrm{p} 53(\mathrm{t}=135$ minutes $)$. By $\mathrm{t}=180$ minutes, the $\mathrm{p} 53$ concentration begins to increase again, giving a period of oscillation of approximately 3 hours.

[Fig. 19 about here.]

Fig. 20 shows the plots of Mdm2 protein concentration over time. Notice that $\mathrm{Mdm} 2$ protein is not produced in significant quantities until around $\mathrm{t}=90$ minutes, reflecting the time for Mdm 2 mRNA production, export from the nucleus and translation in the cytoplasm. Mdm2 levels can then be observed rising in the cytoplasm, beginning to enter the nucleus at $t=135$ minutes, enhancing the degradation of $\mathrm{p} 53$ which in turn causes the down-regulation of Mdm2 expression. This is shown at $\mathrm{t}=225$ minutes where Mdm2 levels have depleted considerably.

[Fig. 20 about here.]

Once again, in order to investigate the influence of spatial effects, we carried out a number of simulations on system (46) - (51), where we varied the values of the diffusion coefficients (all other parameters remaining unchanged) of the mRNAs and proteins. By doing this we found a range of values for the diffusion 
coefficients $D_{c_{1}}, D_{c_{2}}, D_{c_{3}}, D_{n_{1}}, D_{n_{2}}, D_{n_{3}}$ where the system exhibits oscillatory dynamics, i.e., for $7.41 \times 10^{-12} \mathrm{~cm}^{2} \mathrm{~s}^{-1} \leq D_{c}, D_{n} \leq 2.48 \times 10^{-10} \mathrm{~cm}^{2} \mathrm{~s}^{-1}$ oscillations were observed, while no oscillations were observed outside this range. Hence if diffusion of mRNA and protein is either too slow or too fast, oscillations do not occur (as per Hes1 results). In addition to this we calculated the following ranges of degradation rates and Hill coefficients for which, if all other parameters remain unaltered, oscillations are observed: $\mu=0$ to $5.19 \times 10^{-4} \mathrm{~s}^{-1}, v=2.67 \times 10^{-3}$ to $1.56 \mathrm{~s}^{-1}, \rho=2.96 \times 10^{-3}$ to $4.30 \times 10^{-3} \mathrm{~s}^{-1}, \phi=2.59 \times 10^{-4}$ to $1.59 \times 10^{-3} \mathrm{~s}^{-1}$, $h_{1} \geq 2, h_{2} \geq 2$.

Finally, as with the Hes1 system, we carried out a number of numerical simulations of the PDE system (46) - (51) where we investigated the effect of varying the two functions $H_{1}(\bar{x}, \bar{y})$ and $H_{2}(\bar{x}, \bar{y})$, controlling constant protein synthesis and protein translation via mRNA in the cytoplasm, respectively (see Appendix for full details). Once again the simulation results (similar to Figs. 9-11 but not shown here) reveal an "optimum" distance outside the nucleus for protein production for which undamped oscillations of large amplitude are observed.

\section{Discussion}

Dissecting the mechanisms by which transcription factors are regulated within cells is critical to understanding cellular function in health and disease and the opportunities for therapeutic intervention. Results from previous mathematical models have reflected simplified experimental findings but have not distinguished explicitly between spatial compartments within the cell and have not considered (explicitly) spatial movement of molecules. Spatial localisation and considerations are particularly important when modelling transcription factors, which although produced in the cytoplasm must be translocated to the nucleus to function. For the p53 pathway, hindering p53 translocation to the nucleus alters the transcriptome of the cell and contributes to carcinogenesis (Vousden and Prives 2009). Using partial differential equations (PDEs), we can model these aspects of intracellular signalling pathways explicitly.

The simulation results of our models have demonstrated the existence of oscillatory dynamics in negative feedback systems both for relatively simple (Hes1) and more complex (p53-Mdm2) pathways and have been able to focus on reactions occurring both in the cell nucleus and in the cytoplasm. The main advantage of using systems of PDEs to model intracellular reactions is that the PDEs enable spatial effects to be examined explicitly. 
In both the Hes1 and p53-Mdm2 system we varied the diffusion coefficients of the mRNAs and proteins and found a range of values for these diffusion coefficients where the system exhibits oscillatory dynamics, i.e., the results of the model have predicted a range of diffusion coefficients for the molecules involved so that oscillations can be observed. By varying the diffusion coefficients of the molecules, we can vary the flux rates across the nuclear membrane (equivalent to varying nuclear import and export rates), thus granting greater control and allowing a much more in depth analysis of the systems. Similar results were obtained by varying the mRNA degradation rates, protein degradation rates and Hill coefficients, further demonstrating that the oscillations are robust to parameter changes.

Exploiting the explicitly spatial nature of PDEs, we were also able to manipulate mathematically the location of the ribosomes, thus controlling where the proteins were synthesized within the cytoplasm. For both the Hes 1 and the p53Mdm2 systems, we carried out a number of numerical simulations where we investigated the effect of varying the two functions $H_{1}(\bar{x}, \bar{y})$ and $H_{2}(\bar{x}, \bar{y})$, controlling constant protein synthesis and protein translation via mRNA in the cytoplasm, respectively. For both model systems, the simulation results revealed an "optimum" distance outside the nucleus for protein production for which sustained (undamped) oscillations of large amplitude were observed.

Future work in this area will undoubtedly become more accurate and complex, but is likely to help elucidate the complex biochemical pathways involved in cellular decisions to undergo cell cycle arrest, senescence or apoptosis (Vousden and Prives 2009; Toledo and Wahl 2006). In particular, extensions of the current model will consider the active transport of proteins and mRNA within the cell as mechanisms of movement in addition to diffusion (Cangiani and Natalini 2010). We also plan to model the nuclear membrane in more detail and take into account its thickness. This will allow us to model differences in the rate of transport of mRNA and protein across the nuclear membrane more accurately. Additional complexities of post-transcriptional mRNA and post-translational protein modifications, while not explicitly incorporated into the model can easily be done and will be included in future work (Abou-Jaoudé et al. 2009; Zhang et al. 2007).

The spatial models presented here reflect experimental findings both in vitro (Hirata et al. 2002; Geva-Zatorsky et al. 2006) and in vivo (Hamstra et al. 2006) and mark a conceptual advance in the modelling of intracellular processes. With the emergence of new imaging technologies, validation of spatial models will be possible, with dynamic molecular imaging of subcellular processes on the near horizon.

Future models should enable us to drill down into the fundamental differences 
between cancer cells and normal cells. As an exemplar, using the p53-Mdm2 pathway we will be able to model the effects of different therapeutic approaches, including the temporal and spatial distributions of targeted disruption of p53 or Mdm2 interactions by non-genotoxic mechanisms.

\section{Acknowledgments}

The authors gratefully acknowledge the support of the ERC Advanced Investigator Grant 227619, "M5CGS - From Mutations to Metastases: Multiscale Mathematical Modelling of Cancer Growth and Spread".

\section{Appendix: Protein Translation and Synthesis in the Cytoplasm: Considera- tion of the Location of the Endoplasmic Reticulum}

The endoplasmic reticulum (ER) is a network of flattened sacs and branching tubules surrounding the cell nucleus that extends throughout the cytoplasm in eukaryotic cells. These sacs and tubules are all interconnected by a single continuous membrane so that the organelle has only one large and intricately arranged lumen. Proteins made in the endoplasmic reticulum are mainly either exported to the exterior of the cell or are transported to other membrane structures such as the Golgi apparatus, lysosomes and endosomes (Alberts et al. 2008). Thus proteins made in the endoplasmic reticulum are unlikely to return to the nucleus.

In our models of the Hes 1 and p53 pathways, we have made allowance for the endoplasmic reticulum by assuming that proteins made in the cytoplasm are translated a certain distance outside the nucleus. We now state this assumption explicitly using step functions in the relevant equations for the computational cell domain shown in Fig. 21. For the Hes1 system, the equation for Hes1 protein (17) is written as:

$$
\frac{\partial\left[\bar{p}_{c}\right]}{\partial \bar{t}}=D_{p_{c}}^{*} \nabla^{2}\left[\bar{p}_{c}\right]+H_{1}(\bar{x}, \bar{y}) \alpha_{p}^{*}\left[\bar{m}_{c}\right]-\mu_{p}^{*}\left[\bar{p}_{c}\right],
$$

and in the p53-Mdm2 system, we can write the equations for p53 and Mdm2 concentrations in the cytoplasm (46) (50) as:

$$
\begin{aligned}
\frac{\partial\left[\overline{p 53}_{c}\right]}{\partial \bar{t}} & =D_{c_{1}}^{*} \nabla^{2}\left[\overline{p 53}_{c}\right]+H_{2}(\bar{x}, \bar{y}) \beta^{*}-\left(\mu^{*}+v^{*}\left(\frac{\left[\overline{M d m 2}_{c}\right]^{h_{1}}}{M d m 2^{*}+\left[\overline{M d m 2}_{c}\right]^{h_{1}}}\right)\right)\left[\overline{p 53}_{c}\right], \\
\frac{\partial\left[\overline{M d m 2}_{c}\right]}{\partial \bar{t}} & =D_{c_{3}}^{*} \nabla^{2}\left[\overline{M d m 2}_{c}\right]+H_{1}(\bar{x}, \bar{y}) \gamma^{*}\left[{\overline{M d m 2 m_{c}}}_{c}\right]-\rho^{*}\left[\overline{M d m 2}_{c}\right],
\end{aligned}
$$


where the functions $H_{1}(\bar{x}, \bar{y})$ and $H_{2}(\bar{x}, \bar{y})$ are defined as:

$$
H_{1}(\bar{x}, \bar{y})= \begin{cases}0, & \text { if } \frac{\bar{x}^{2}}{2}+\bar{y}^{2} \leq 0.25, \\ 1, & \text { if } \frac{\bar{x}^{2}}{2}+\bar{y}^{2}>0.25\end{cases}
$$

and

$$
H_{2}(\bar{x}, \bar{y})= \begin{cases}0, & \text { if } \frac{\bar{x}^{2}}{2}+\bar{y}^{2} \leq 0.25 \\ 1, & \text { if } 0.25<\frac{\bar{x}^{2}}{2}+\bar{y}^{2}<0.375 \\ 0, & \text { if } \frac{\bar{x}^{2}}{2}+\bar{y}^{2} \geq 0.375\end{cases}
$$

The function $H_{1}(\bar{x}, \bar{y})$ is such that in a region close to the nucleus (representing the location of the ER), the function is zero, meaning there is no protein synthesis in this region. In a region further away from the nucleus (outside the ER) the function takes the value of one, modelling the translation of protein in this region of the cytoplasm. The function $H_{2}(\bar{x}, \bar{y})$ is such that in a region close to the nucleus the function is zero, meaning there is no protein synthesis here. However, it is now assumed that the function takes the value of one in an annular region outside of the ER (again modelling the translation of protein). An annular region is chosen because we assume p53 is produced at a constant rate in the cytoplasm. This prevents p53 from being produced close to the plasma membrane, where mRNA is unlikely to reach in sufficient quantities. The two functions are illustrated graphically in Fig. 21.

[Fig. 21 about here.] 


\section{References}

W. Abou-Jaoudé, D.A. Ouattara, and M. Kaufman. From structure to dynamics: Frequency tuning in the p53-Mdm2 network i. Logical approach. J. Theor. Biol., 258:561-577, 2009.

S. Agrawal, C. Archer, and D.V. Schaffer. Computational models of the notch network elucidate mechanisms of context-dependent signaling. PLoS Comput Biol., 5:e1000390, 2009.

B. Alberts, A. Johnson, J. Lewis, M. Raff, K. Roberts, and P. Walter. Molecular Biology of the Cell. Garland Science, Taylor and Francis Group Ltd, Oxford, fifth edition, 2008.

R.L. Bar-Or, R. Maya, L.A. Segel, U. Alon, A.J. Levine, and M. Oren. Generation of oscillations by the p53-Mdm2 feedback loop: A theoretical and experimental study. PNAS, 97:11250-11255, 2000.

E. Batchelor, C.S. Mock, I. Bhan, A. Loewer, and G. Lahav. Recurrent initiation: A mechanism for triggering p53 pulses in response to DNA damage. Mol. Cell., 30:277-289, 2008.

W.P. Bennet, S.P. Hussain, K.H. Vahakangas, M.A. Khan, P.G. Shields, and C.C. Harris. Molecular epidemiology of human cancer risk: gene environment interactions and p53 mutation spectrum in human lung cancer. J. Pathol, 187:8-18, 1999.

S. Busenberg and J.M. Mahaffy. Interaction of spatial diffusion and delays in models of genetic control by repression. J. Math. Biol., 22:313-333, 1985.

A. Cangiani and R. Natalini. A spatial model of cellular molecular trafficking including active transport along microtubules. J. Theor. Biol., 267:614-625, 2010 .

S. Carter and K.H. Vousden. Modifications of p53: competing for the lysines. Curr. Opin. Genet. Dev., 19:18-24, 2009.

A. Ciliberto, B. Novak, and J.J. Tyson. Steady states and oscillations in the p53/Mdm2 network. Cell Cycle, 4:488-493, 2005.

A.S. Coutts, C.J. Adams, and N.B. La Thangue. p53 ubiquitination by Mdm2: a never ending tail? DNA Repair (Amst)., 8:483-490, 2009. 
R. Montes de Oca Luna, D.S. Wagner, and G. Lozano. Rescue of early embryonic lethality in mdm2-deficient mice by deletion of p53. Nature, 378:203-206, 1995.

C.P. Fall, E.S. Marland, J.M. Wagner, and J.J. Tyson. Computational Cell Biology. Springer, New York, fifth edition, 2002.

N. Geva-Zatorsky, N. Rosenfeld, S. Itzkovitz, R. Milo, A. Sigal, E. Dekel, T. Yarnitzky, Y. Liron, P. Polak, G. Lahav, and U. Alon. Oscillations and variability in the p53 system. Mol. Syst. Biol., 2:E1-E13, 2006.

B.C. Goodwin. Oscillatory behavior in enzymatic control processes. Adv. Enzyme Regul., 3:425-428, 1965.

K.E. Gordon, I.M.M. Van Leeuwen, S. Laín, and M.A.J. Chaplain. Spatiotemporal modelling of the p53-mdm2 oscillatory system. Math. Model. Nat. Phenom., 4:97-116, 2009.

D.A. Hamstra, M.S. Bhojani, L.B. Griffin, B. Laxman, B.D. Ross, and A. Rehemtulla. Real-time evaluation of $\mathrm{p} 53$ oscillatory behaviour in vivo using bioluminescent imaging. Cancer Res., 66:7482-7489, 2006.

H. Hirata, S. Yoshiura, T. Ohtsuka, Y. Bessho, T. Harada, K.Yoshikawa, and R. Kageyama. Oscillatory expression of the bHLH factor Hes1 regulated by a negative feedback loop. Science, 298:840-843, 2002.

S.N. Jones, A.E. Roe, L.A. Donehower, and A. Bradley. Rescue of embryonic lethality in Mdm2-deficient mice by absence of p53. Nature, 378:206-208, 1995.

N. Klonis, M. Rug, I. Harper, M. Wickham, A. Cowman, and L. Tilley. Fluorescence photobleaching analysis for the study of cellular dynamics. Eur. Biophys. $J ., 31: 36-51,2002$.

G. Lahav, N. Rosenfeld, A. Sigal, N. Geva-Zatorsky, A.J. Levine, M.B. Elowitz, and U. Alon. Dynamics of the p53-Mdm2 feedback loop in individual cells. Nat. Genet., 36:147-150, 2004.

J. Lewis. Autoinhibition with transcriptional delay: a simple mechanism for the zebrafish somitogenesis oscillator. Curr. Biol., 13:1398-1408, 2003. 
L. Ma, J. Wagner, J.J. Rice, W. Hu, A.J. Levine, and G.A. Stolovitzky. A plausible model for the digital response of p53 to DNA damage. PNAS, 102:1426614271, 2005.

J.M. Mahaffy. Genetic control models with diffusion and delays. Math. Biosci., 90:519-533, 1988.

J.M. Mahaffy and C.V. Pao. Models of genetic control by repression with time delays and spatial effects. J. Math. Biol., 20:39-57, 1984.

J.C. Marine and A.G. Jochemsen. Mdmx and Mdm2: brothers in arms? Cell Cycle, 3:900-904, 2003.

T. Matsuda, A. Miyawaki, and T. Nagai. Direct measurement of protein dynamics inside cells using a rationally designed photoconvertible protein. Nat. Methods, 5:339-345, 2008.

G.I. Mihalas, M. Neamtu, D. Opris, and R.F. Horhat. A dynamic P53-MDM2 model with time delay. Chaos, Solitons and Fractals, 30:936-945, 2006.

H. Momiji and N.A.M. Monk. Dissecting the dynamics of the Hes1 genetic oscillator. J. Theor. Biol., 254:784-798, 2008.

N.A.M. Monk. Oscillatory expression of Hes1, p53, and NF- $\kappa \mathrm{B}$ driven by transcriptional time delays. Curr. Biol., 13:1409-1413, 2003.

D.E. Nelson, A.E.C. Ihekwaba, M. Elliott, J.R. Johnson, C.A. Gibney, B.E. Foreman, G. Nelson, V. See, C.A. Horton, D.G. Spiller, S.W. Edwards, H.P. McDowell, J.F. Unitt, E. Sullivan, R. Grimley, N. Benson, D. Broomhead, D.B. Kell, and M.R.H. White. Oscillations in NF- $\kappa \mathrm{B}$ signaling control the dynamics of gene expression. Science, 306:704-708, 2004.

D.A. Ouattara, W. Abou-Jaoudé, and M. Kaufman. From structure to dynamics: Frequency tuning in the p53-Mdm2 network. ii Differential and stochastic approaches. J. Theor. Biol., 264:1177-1189, 2010.

C.J. Proctor and D.A. Gray. Explaining oscillations and variability in the p53Mdm2 system. BMC Syst. Biol., 2(75):1-20, 2008. doi: 10.1186/1752-0509-275 .

K. Puszyński, B. Hat, and T. Lipniacki. Oscillations and bistability in the stochastic model of p53 regulation. J. Theor. Biol., 254:452-465, 2008. 
O. Seksek, J. Biwersi, and A.S. Verkman. Translational diffusion of macromolecule-sized solutes in cytoplasm and nucleus. J. Cell. Biol., 138: 131-142, 1997.

H. Shankaran, D.L. Ippolito, W.B. Chrisler, H. Resat, N. Bollinger, L.K. Opresko, and H.S. Wiley. Rapid and sustained nuclear-cytoplasmic ERK oscillations induced by epidermal growth factor. Mol. Syst. Biol., 5:322, 2009.

J.M. Stommel and G.M. Wahl. Accelerated MDM2 auto-degradation induced by DNA-damage kinases is required for p53 activation. Embo. J., 23:1574-1556, 2004.

F. Toledo and G.M. Wahl. Regulating the $\mathrm{p} 53$ pathway: in vitro hypotheses, in vivo veritas. Nat. Rev. Cancer, 6:909-923, 2006.

B. Vogelstein, D. Lane, and A.J. Levine. Surfing the p53 network. Nature, 408: 307-310, 2000.

K.H. Vousden and C. Prives. Blinded by the light: the growing complexity of p53. Cell, 137:413-431, 2009.

D.P. Xirodimas, C.W. Stephen, and D.P. Lane. Cocompartmentalization of p53 and Mdm2 is a major determinant for Mdm2-mediated degradation of p53. Exp. Cell. Res., 270:66-77, 2001.

T. Zhang, P. Brazhnik, and J.J. Tyson. Exploring mechanisms of the DNA-damage response: p53 pulses and their possible relevance to apoptosis. Cell Cycle, 6: 85-94, 2007.

J.T. Zilfou and S.W. Lowe. Tumor suppressive functions of p53. Cold Spring Harb Perspect Biol., 1:a001883, 2009. 


\section{List of Figures}

1 A generic negative feedback loop. . . . . . . . . . . . 33

2 A schematic representation of the Hes1 model. hes1 mRNA is transcribed in the nucleus. It is then exported to the cytoplasm where translation into Hes1 protein occurs. hes1 mRNA is then inhibited in the nucleus by its own protein. This is one of the simplest examples of a negative feedback loop. . . . . . . . . 34

3 Schematic diagram showing how the spatial interactions between hes 1 mRNA and Hes 1 protein are modelled. hes 1 mRNA is produced in the nucleus (transcription), then exported across the nuclear membrane into the cytoplasm where it is translated into protein, i.e., transcription occurs exclusively in the nucleus and translation/synthesis occurs exclusively in the cytoplasm. Hes 1 protein is then imported back across the nuclear membrane to the nucleus where it inhibits the production of its own mRNA, i.e., a negative feedback loop exists. . . . . . . . . . . . . . . 35

4 Plot of the concentrations of hes 1 mRNA (red) and Hes1 protein (blue) in the nucleus over time. The period of oscillations is approximately 120 minutes. Parameter values as per (25). . . . . . 36

5 Plot of the concentrations of hes1 mRNA (red) and Hes1 protein (blue) in the cytoplasm over time. The period of oscillations is approximately 120 minutes. Parameter values as per (25). . . . .

6 Plots showing the spatio-temporal evolution of hes $1 \mathrm{mRNA}$ concentration within the cell from times $t=0$ to $t=480$ minutes at 60 minute intervals. The concentration oscillates in both time and space. Parameter values as per (25). . . . . . . . . . . 38

7 Plots showing the spatio-temporal evolution of Hes1 protein concentration within the cell from times $\mathrm{t}=0$ to $\mathrm{t}=480$ minutes. The concentration oscillates in both time and space. Parameter values as per (25). . . . . . . . . . . . . . . . .

8 Plot showing a snapshot of the Hes 1 protein concentration at $\mathrm{t}=$ 60 minutes in a 3D spherical (a) and ellipsoidal (b) domain. Axes are presented in nondimensional units. Parameter values as per (25). 40 
9 Plots showing the effect on the concentrations of hes 1 mRNA (red) and Hes1 protein (blue) in the nucleus over time by varying the function $H_{1}(\bar{x}, \bar{y})$ which controls the spatial production of protein in the cytoplasm (all other parameters as per (25)). It can be observed from the plots that there exists a range of distances for protein production to begin outside the nucleus in order to see oscillatory dynamics. . . . . . . . . . . . . . . . . 41

10 Plots showing the effect on the concentrations of hes1 mRNA (red) and Hes1 protein (blue) in the cytoplasm over time by varying the function $H_{1}(\bar{x}, \bar{y})$ which controls the spatial production of protein in the cytoplasm (all other parameters as per (25)). It can be observed from the plots that there exists a range of distances for protein production to begin outside the nucleus in order to see oscillatory dynamics. . . . . . . . . . . . . .

11 Plots showing the spatial distribution of Hes1 protein concentration at $\mathrm{t}=60$ minutes for various functions $H_{1}(\bar{x}, \bar{y})$ which controls the spatial production of protein in the cytoplasm (all other parameter values as per (25)). It can be observed from the plots that the protein concentration throughout the cell varies with the function $H_{1}(\bar{x}, \bar{y}) \ldots \ldots \ldots \ldots \ldots \ldots$

12 Plot of the total concentrations of hes 1 mRNA (red) and Hes1 protein (blue) in the nucleus over time when the proteasome is inhibited. No oscillations are observed. Parameter values as per

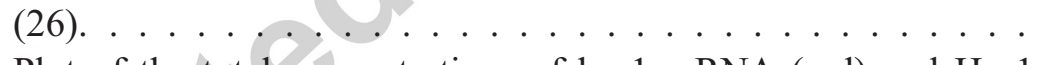

13 Plot of the total concentrations of hes 1 mRNA (red) and Hes1 protein (blue) in the cytoplasm over time when the proteasome is inhibited. No oscillations are observed. Parameter values as per (26) . . . . . . . . . . . . . . .

14 Plots showing the spatio-temporal evolution of hes 1 mRNA concentration within the cell from times $t=0$ to 300 minutes when the proteasome is inhibited. No oscillations are observed. hes1 mRNA levels peak at $\mathrm{t}=60$ minutes and then decrease by $\mathrm{t}=120$ minutes and remain low until the end of the simulation. Parameter values as $\operatorname{per}(26) \ldots \ldots \ldots \ldots$. . . . . . . . . . . . 46 
15 Plots showing the spatio-temporal evolution of Hes1 protein concentration within the cell from times $t=0$ to 300 minutes when the proteasome is inhibited. No oscillations are observed. As expected by decreasing the protein degradation rate, Hes1 protein rises to a high level. It reaches a steady state level by $\mathrm{t}=300$. Parameter values as per (26). . . . . . . . . . . . . . . 47

16 A schematic representation of the p53-Mdm2 pathway. p53 is synthesised in the cytoplasm at a constant rate. It then translocates into the nucleus where it acts as a transcription factor, upregulating Mdm2 mRNA transcription. Mdm2 mRNA is then exported to the cytoplasm where it is translated into $\mathrm{Mdm} 2$ protein. $\mathrm{Mdm} 2$ protein ubiquitinates $\mathrm{p} 53$ in both the nucleus and cytoplasm which results in an increased decay rate for p53. . . . . . . . . . . 48

17 Plots showing the concentrations of p53 (blue), Mdm2 mRNA (green) and Mdm2 (red) in the nucleus. The period of oscillations is approximately 180 minutes. Parameter values as per (60). . . .

18 Plots showing the concentrations of p53 (blue), Mdm2 mRNA (green) and Mdm2 (red) in the cytoplasm. The period of oscillations is approximately 180 minutes. Parameter values as per (60). 50

19 Plots showing the spatio-temporal evolution of $\mathrm{p} 53$ protein concentration within the cell from times $\mathrm{t}=0$ to $\mathrm{t}=360$ minutes. The concentration oscillates in both time and space. The patterns of oscillations bear a striking resemblance to those obtained in individual cells in Geva-Zatorsky et al. (2006). Parameter values as per (60). . . . . . . . . . . . . . . .

20 Plots showing the spatio-temporal evolution of Mdm2 protein concentration within the cell from times $\mathrm{t}=0$ to $\mathrm{t}=360$ minutes. The concentration oscillates in both time and space. Parameter values

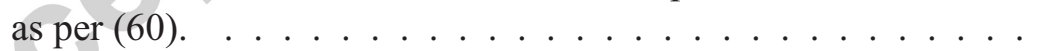

21 Schematic diagram showing the regions where the two functions $H_{1}(\bar{x}, \bar{y})$ and $H_{2}(\bar{x}, \bar{y})$ are non-zero. The blue region of the cytoplasm depicts where we allow constant protein synthesis to occur, i.e., this represents the rectangular function $\mathrm{H}_{2}(\bar{x}, \bar{y})$. The blue and red regions together depict where we allow protein translation via mRNA, i.e., this represents the function $H_{1}(\bar{x}, \bar{y})$. In the white region representing the ER and nucleus, no protein synthesis takes place. ....................... 53 


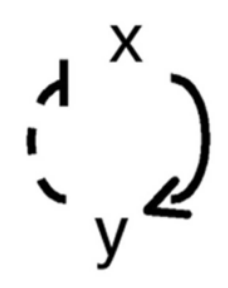

Fig. 1: A generic negative feedback loop. 


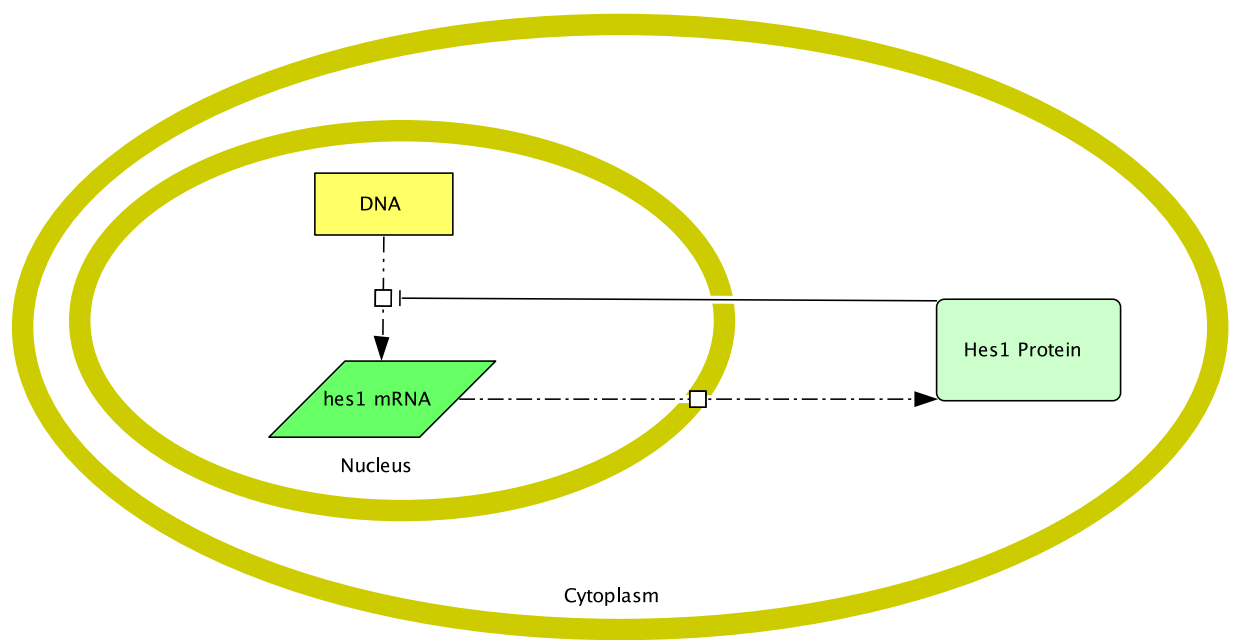

Fig. 2: A schematic representation of the Hes1 model. hes1 mRNA is transcribed in the nucleus. It is then exported to the cytoplasm where translation into Hes1 protein occurs. hes 1 mRNA is then inhibited in the nucleus by its own protein. This is one of the simplest examples of a negative feedback loop. 


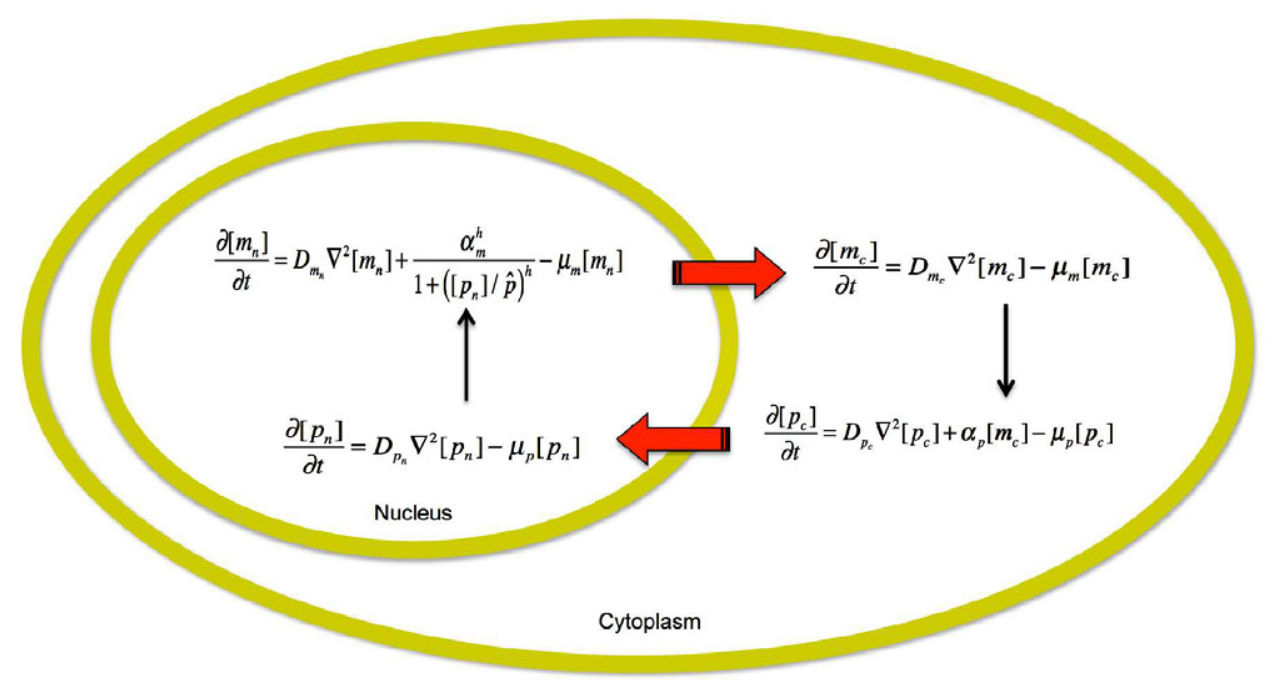

Fig. 3: Schematic diagram showing how the spatial interactions between hes1 mRNA and Hes 1 protein are modelled. hes1 mRNA is produced in the nucleus (transcription), then exported across the nuclear membrane into the cytoplasm where it is translated into protein, i.e., transcription occurs exclusively in the nucleus and translation/synthesis occurs exclusively in the cytoplasm. Hes1 protein is then imported back across the nuclear membrane to the nucleus where it inhibits the production of its own mRNA, i.e., a negative feedback loop exists. 


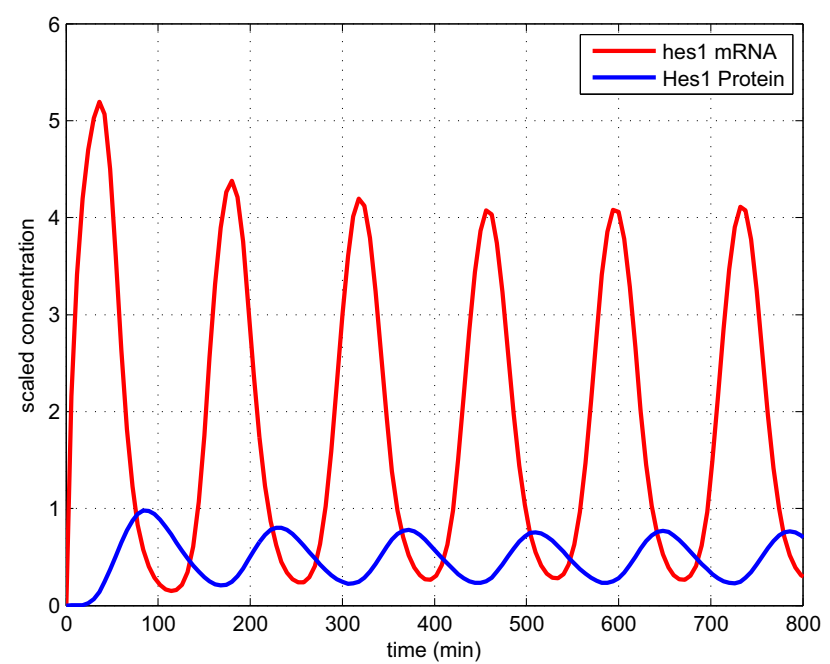

Fig. 4: Plot of the concentrations of hes1 mRNA (red) and Hes 1 protein (blue) in the nucleus over time. The period of oscillations is approximately 120 minutes. Parameter values as per (25). 


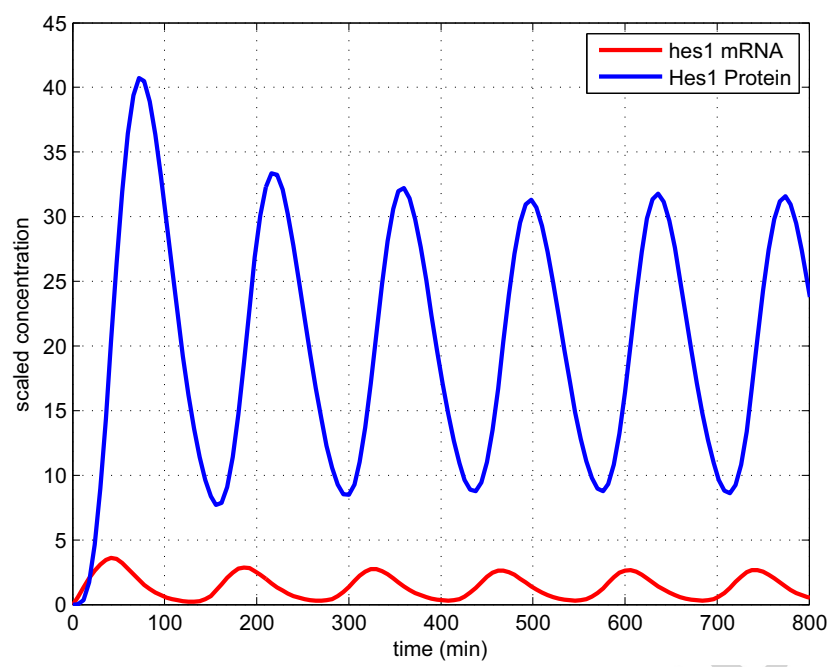

Fig. 5: Plot of the concentrations of hes 1 mRNA (red) and Hes1 protein (blue) in the cytoplasm over time. The period of oscillations is approximately 120 minutes. Parameter values as per (25). 

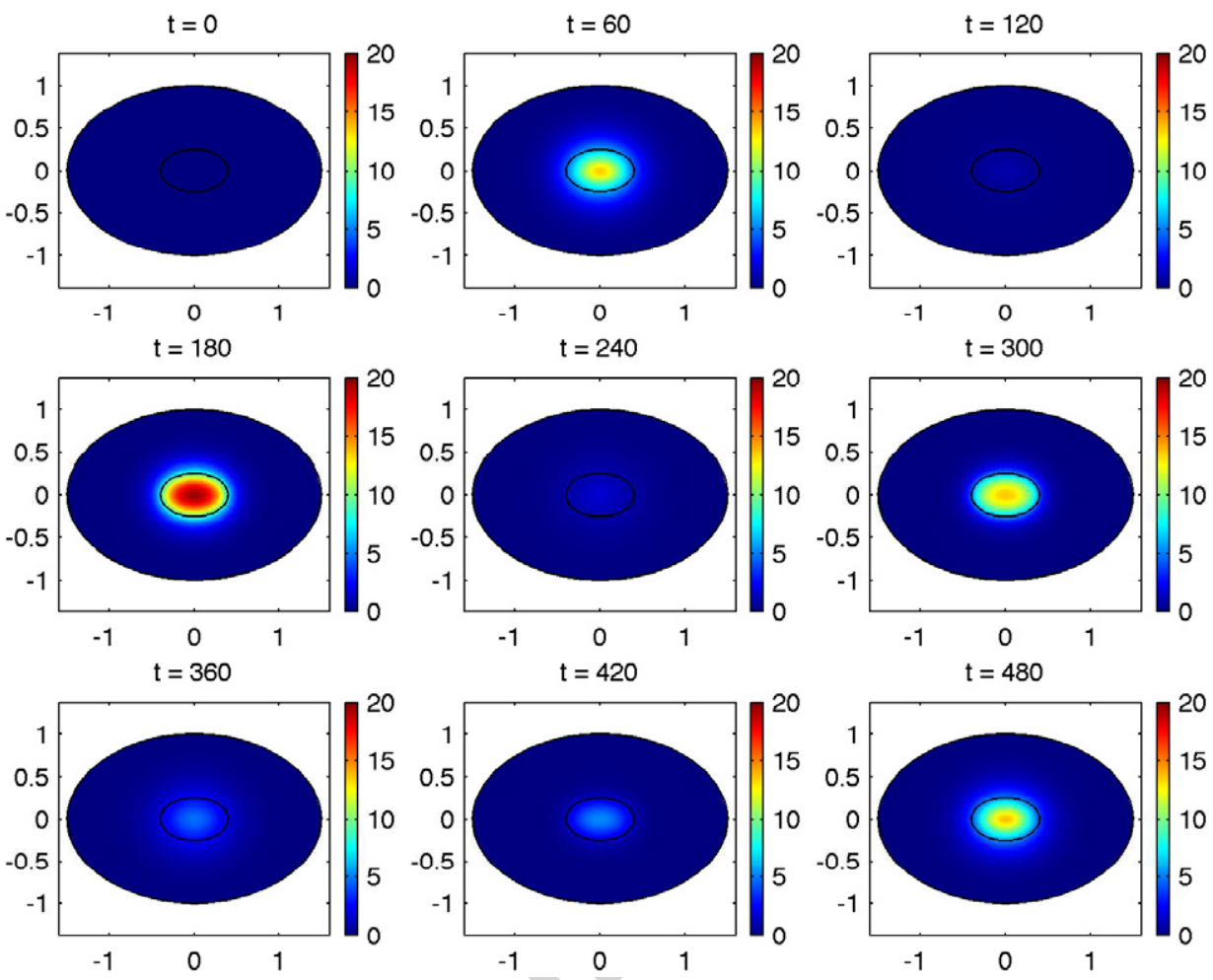

Fig. 6: Plots showing the spatio-temporal evolution of hes 1 mRNA concentration within the cell from times $\mathrm{t}=0$ to $\mathrm{t}=480$ minutes at 60 minute intervals. The concentration oscillates in both time and space. Parameter values as per (25). 

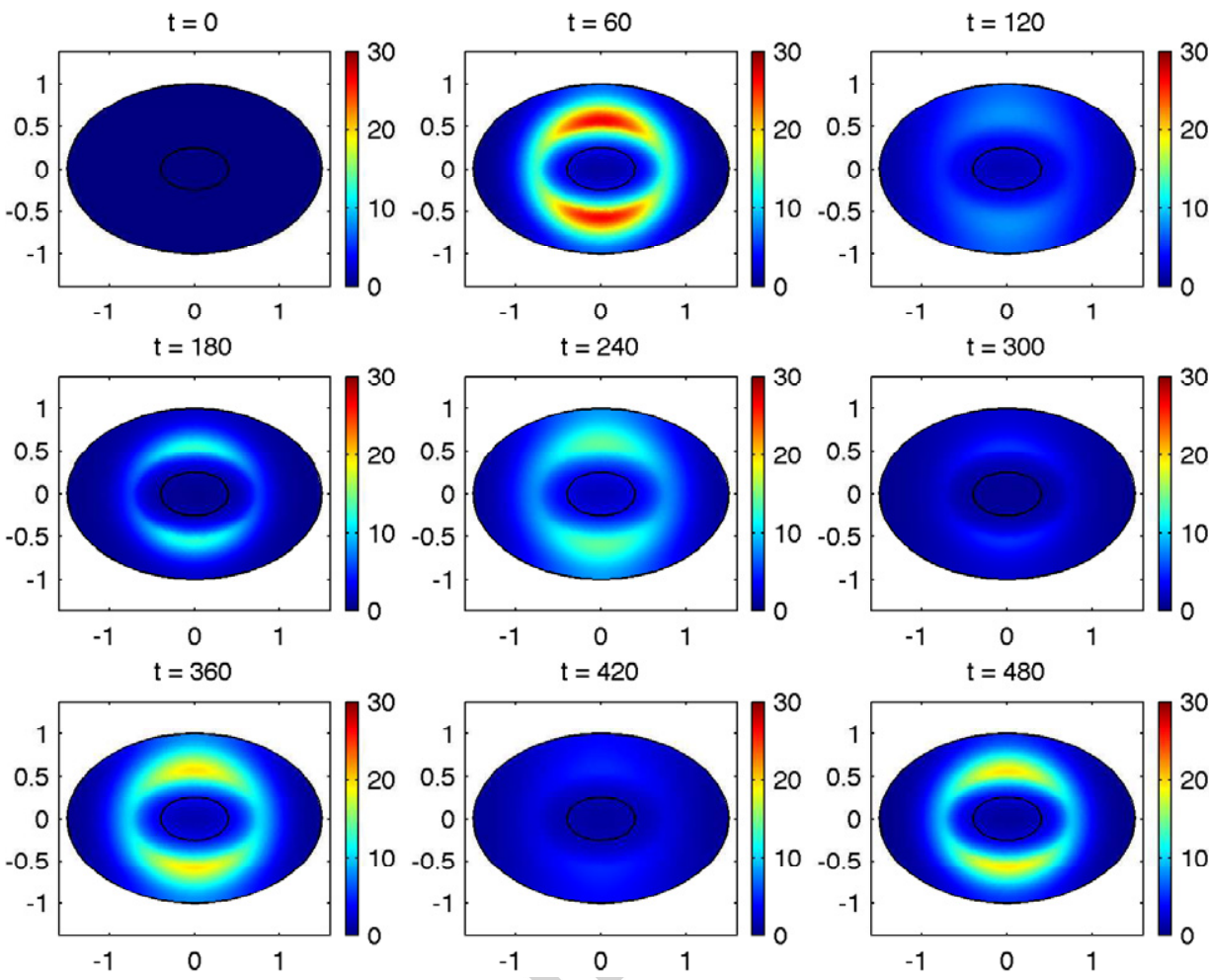

Fig. 7: Plots showing the spatio-temporal evolution of Hes1 protein concentration within the cell from times $t=0$ to $t=480$ minutes. The concentration oscillates in both time and space. Parameter values as per (25). 


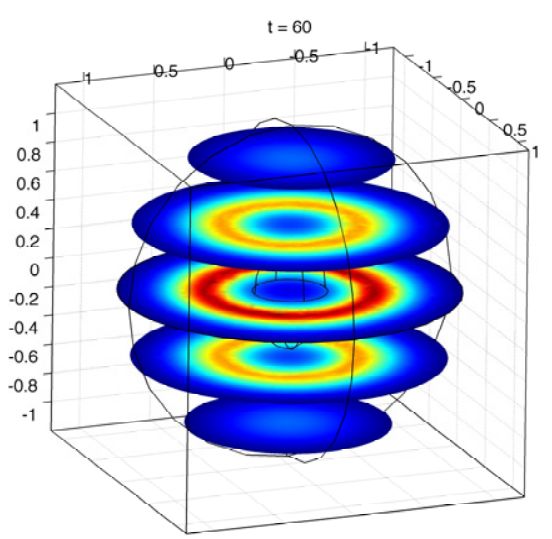

(a) Spherical domain

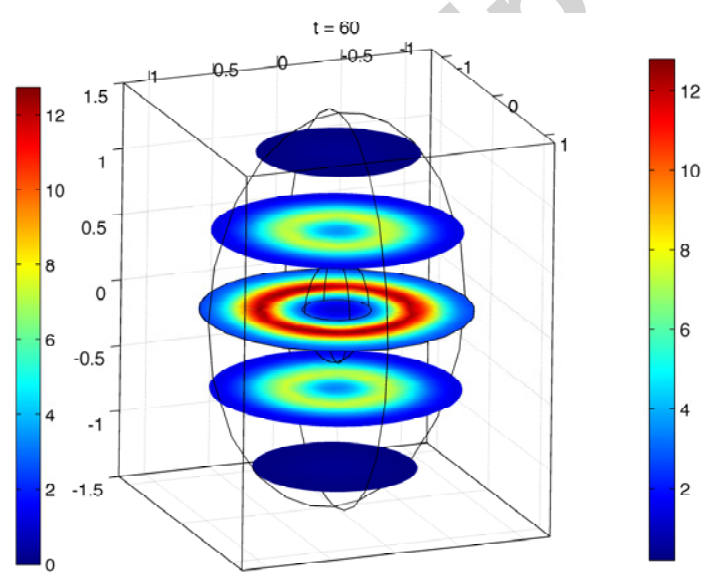

(b) Ellipsoidal domain

Fig. 8: Plot showing a snapshot of the Hes 1 protein concentration at $\mathrm{t}=60$ minutes in a 3D spherical (a) and ellipsoidal (b) domain. Axes are presented in nondimensional units. Parameter values as per (25). 

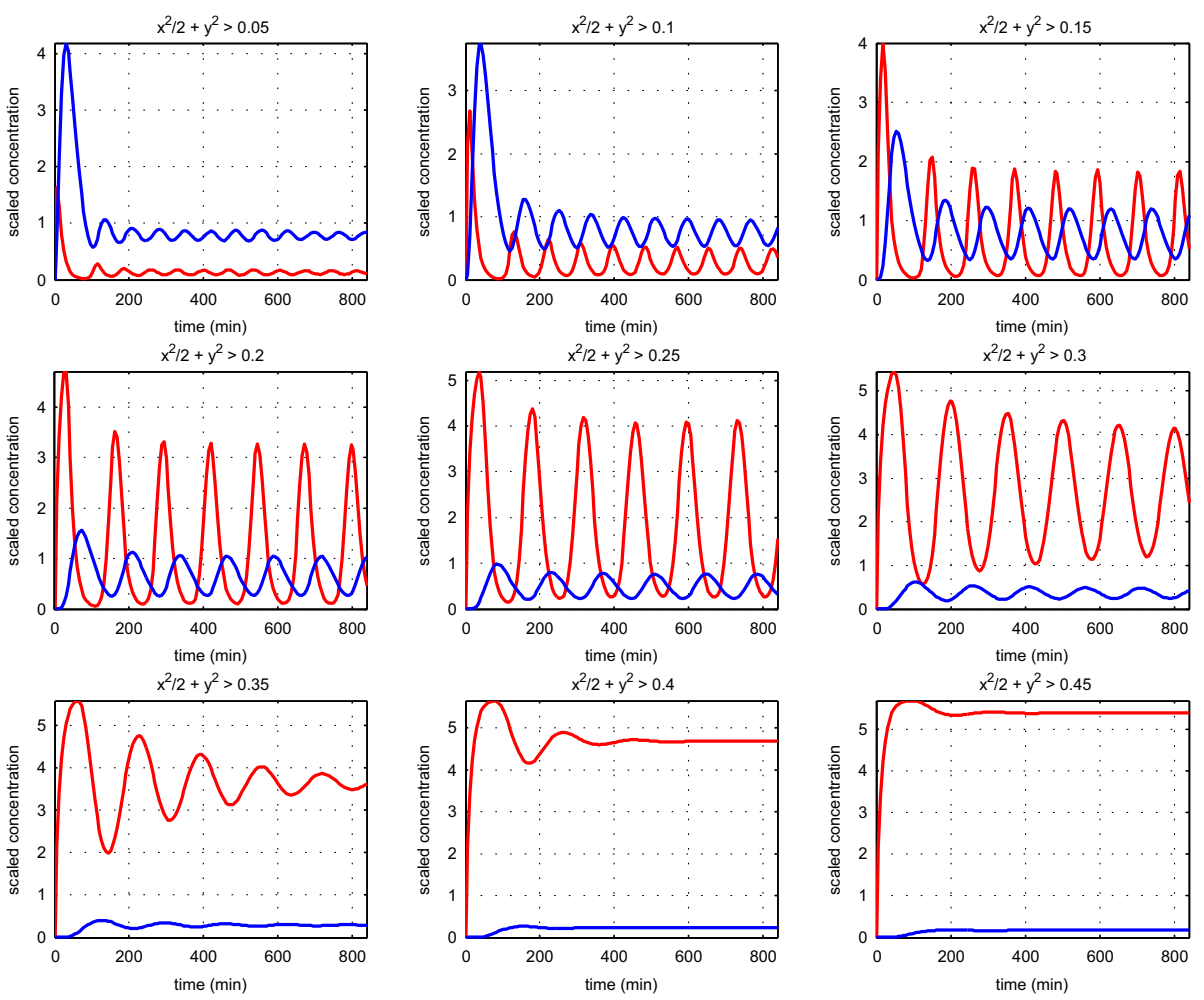

Fig. 9: Plots showing the effect on the concentrations of hes1 mRNA (red) and Hes 1 protein (blue) in the nucleus over time by varying the function $H_{1}(\bar{x}, \bar{y})$ which controls the spatial production of protein in the cytoplasm (all other parameters as per (25)). It can be observed from the plots that there exists a range of distances for protein production to begin outside the nucleus in order to see oscillatory dynamics. 

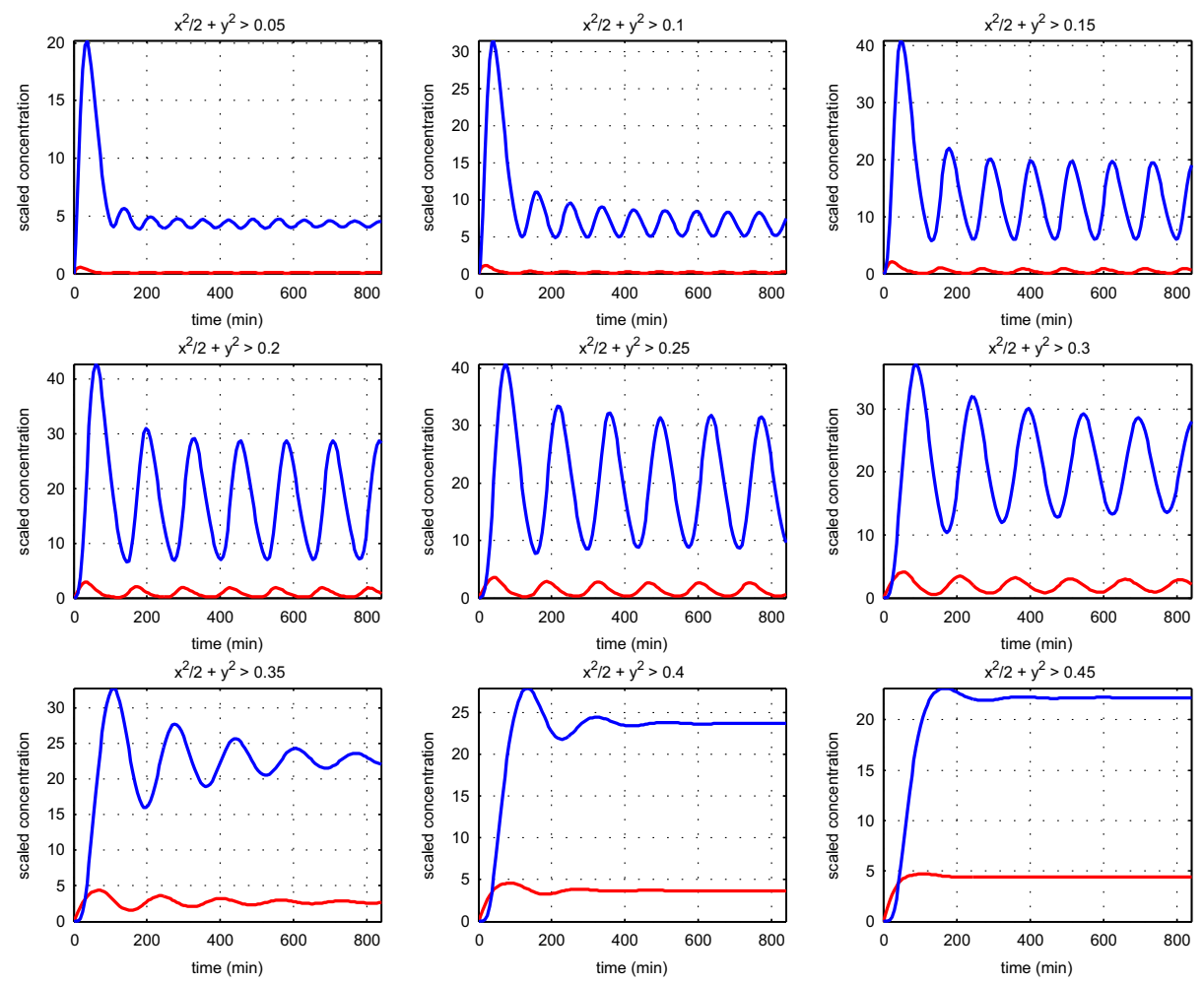

Fig. 10: Plots showing the effect on the concentrations of hes 1 mRNA (red) and Hes1 protein (blue) in the cytoplasm over time by varying the function $H_{1}(\bar{x}, \bar{y})$ which controls the spatial production of protein in the cytoplasm (all other parameters as per (25)). It can be observed from the plots that there exists a range of distances for protein production to begin outside the nucleus in order to see oscillatory dynamics. 

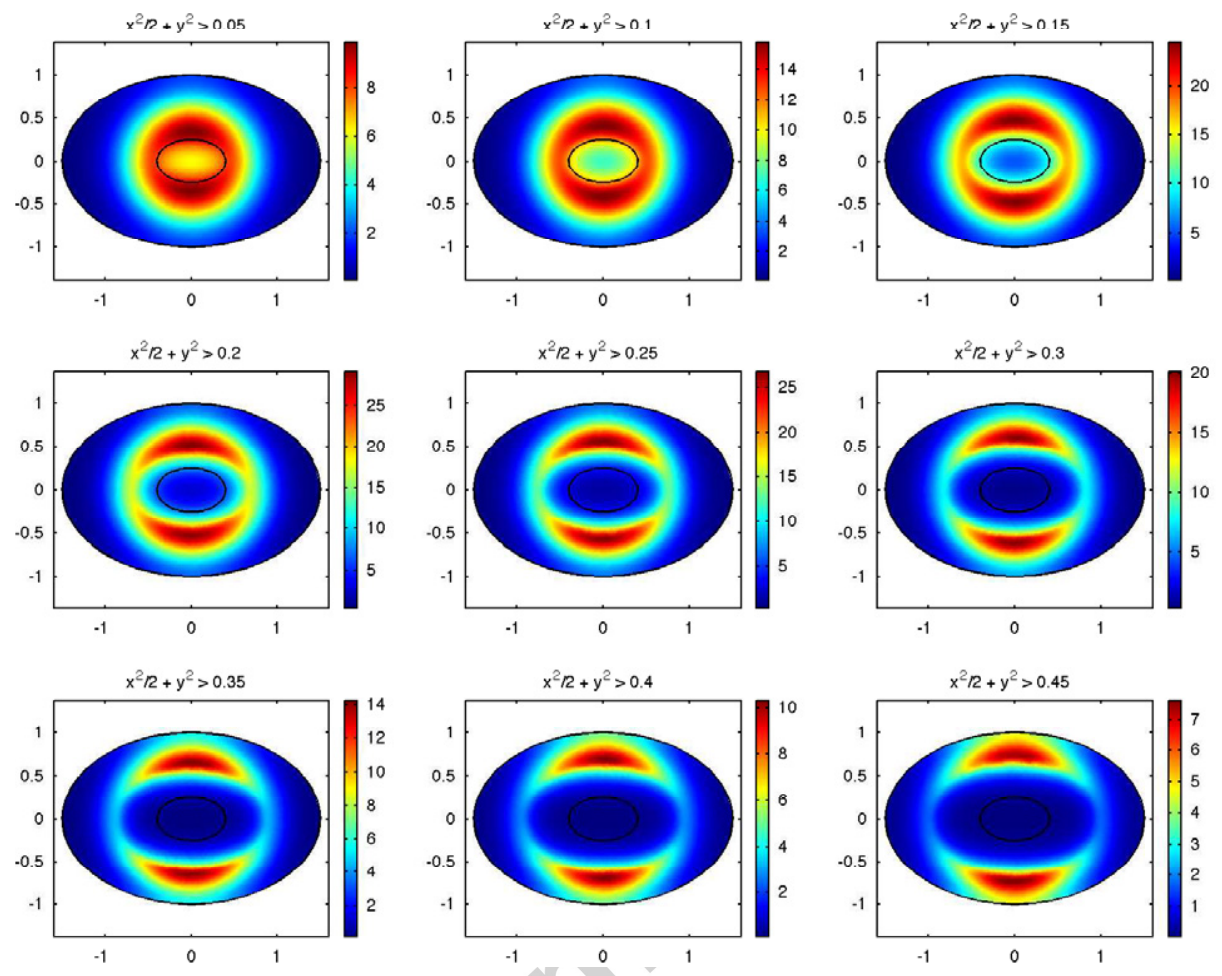

Fig. 11: Plots showing the spatial distribution of Hes1 protein concentration at $\mathrm{t}$ $=60$ minutes for various functions $H_{1}(\bar{x}, \bar{y})$ which controls the spatial production of protein in the cytoplasm (all other parameter values as per (25)). It can be observed from the plots that the protein concentration throughout the cell varies with the function $H_{1}(\bar{x}, \bar{y})$. 


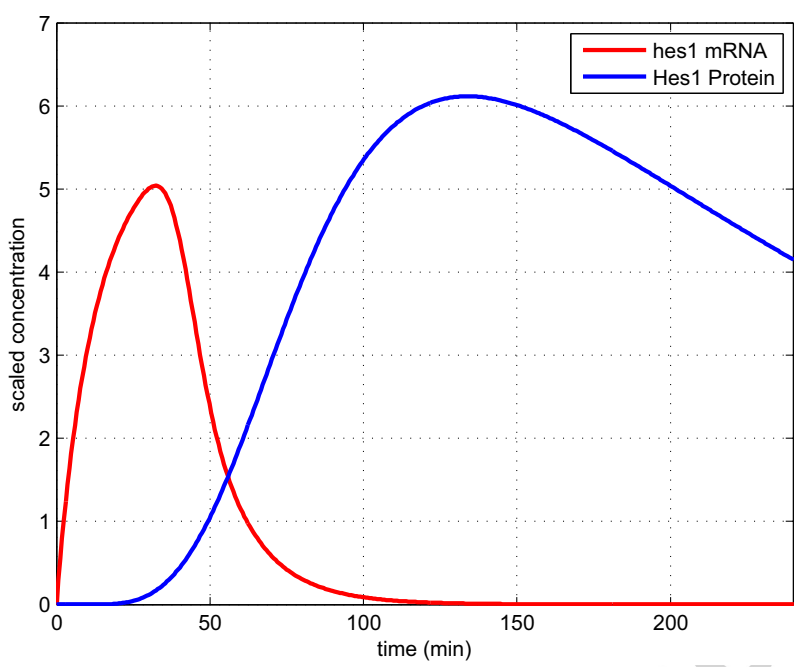

Fig. 12: Plot of the total concentrations of hes1 mRNA (red) and Hes1 protein (blue) in the nucleus over time when the proteasome is inhibited. No oscillations are observed. Parameter values as per (26). 


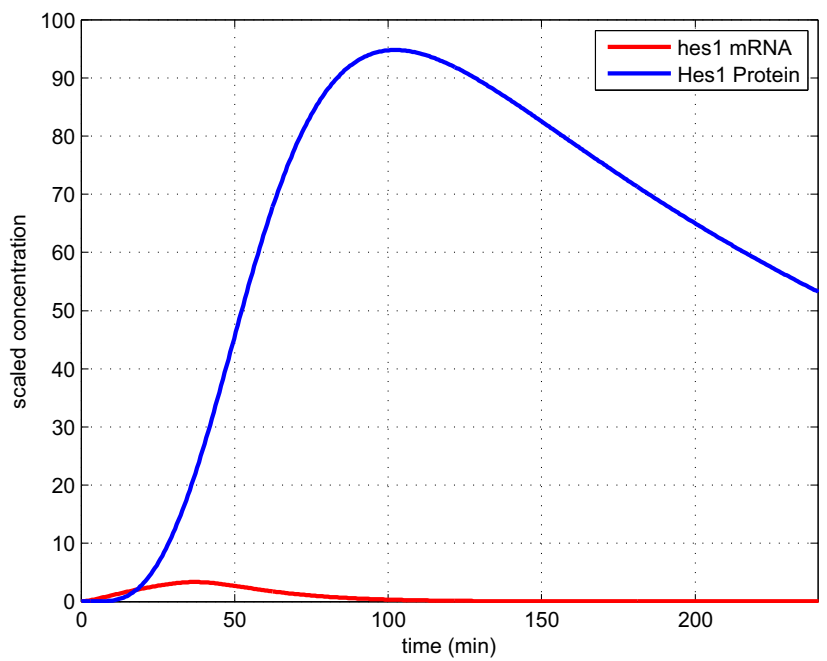

Fig. 13: Plot of the total concentrations of hes1 mRNA (red) and Hes1 protein (blue) in the cytoplasm over time when the proteasome is inhibited. No oscillations are observed. Parameter values as per (26). 

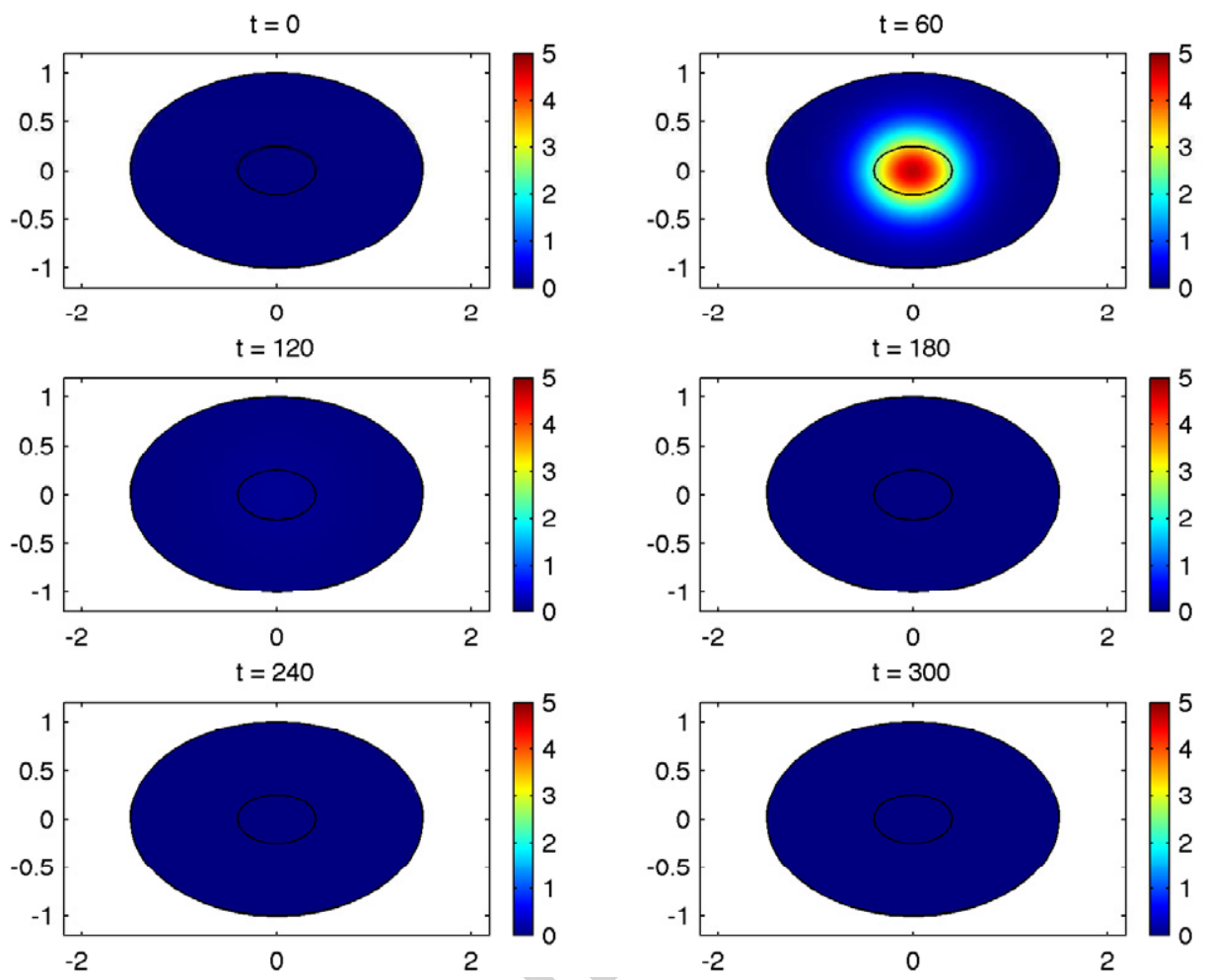

Fig. 14: Plots showing the spatio-temporal evolution of hes 1 mRNA concentration within the cell from times $t=0$ to 300 minutes when the proteasome is inhibited. No oscillations are observed. hes 1 mRNA levels peak at $t=60$ minutes and then decrease by $\mathrm{t}=120$ minutes and remain low until the end of the simulation. Parameter values as per (26). 

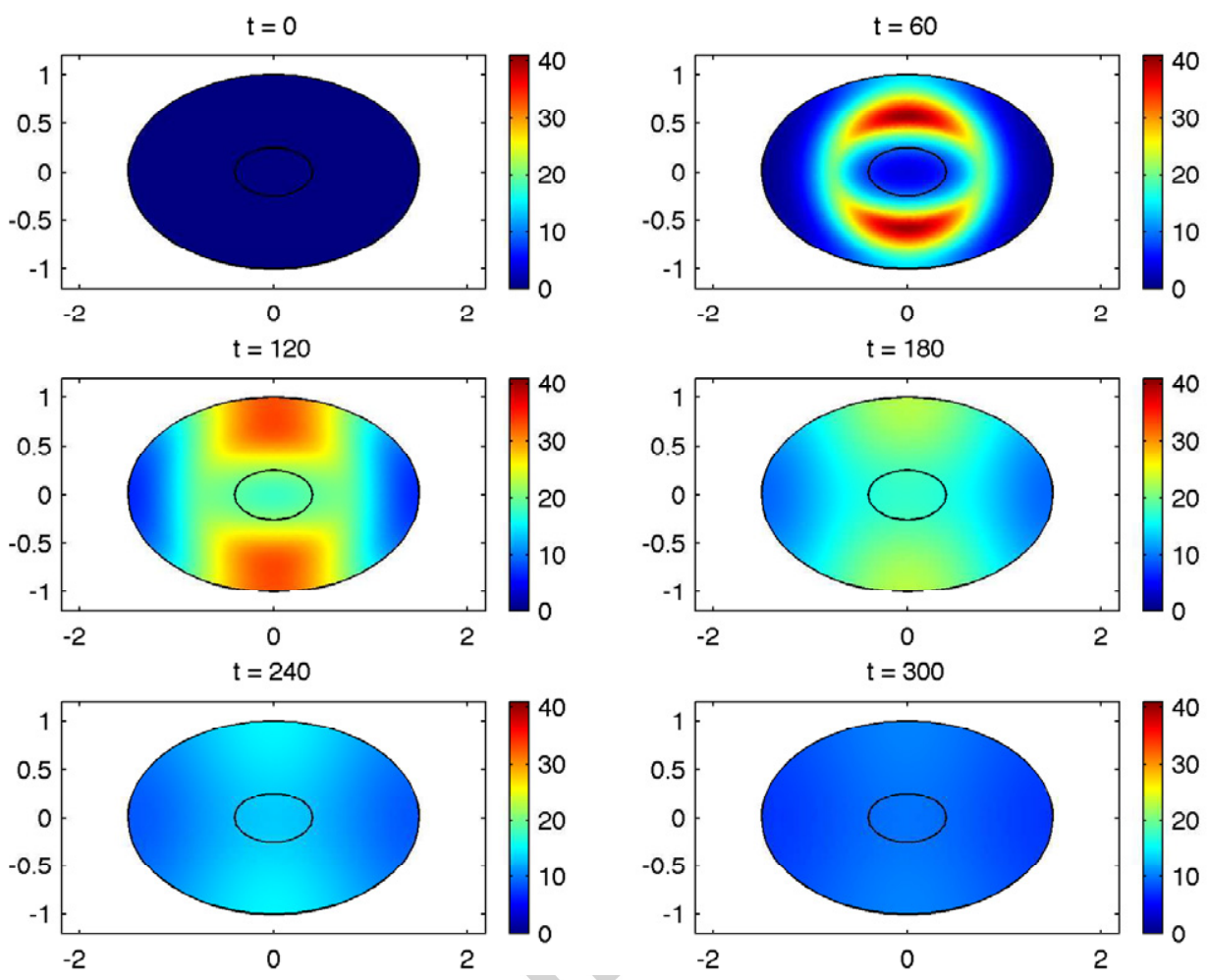

Fig. 15: Plots showing the spatio-temporal evolution of Hes 1 protein concentration within the cell from times $t=0$ to 300 minutes when the proteasome is inhibited. No oscillations are observed. As expected by decreasing the protein degradation rate, Hes1 protein rises to a high level. It reaches a steady state level by $\mathrm{t}=300$. Parameter values as per (26). 


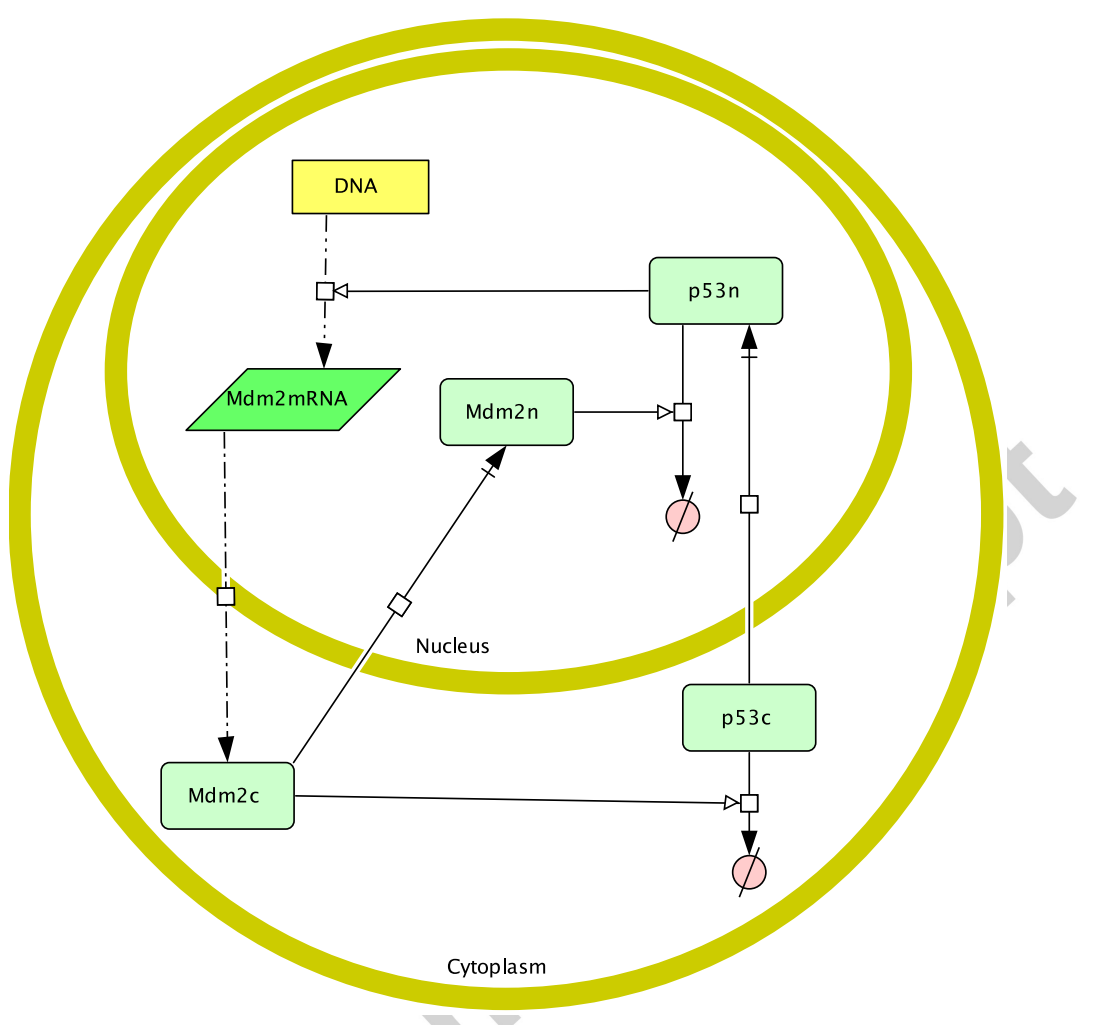

Fig. 16: A schematic representation of the $\mathrm{p} 53-\mathrm{Mdm} 2$ pathway. $\mathrm{p} 53$ is synthesised in the cytoplasm at a constant rate. It then translocates into the nucleus where it acts as a transcription factor, upregulating Mdm2 mRNA transcription. Mdm2 mRNA is then exported to the cytoplasm where it is translated into Mdm2 protein. Mdm2 protein ubiquitinates $\mathrm{p} 53$ in both the nucleus and cytoplasm which results in an increased decay rate for $\mathrm{p} 53$. 


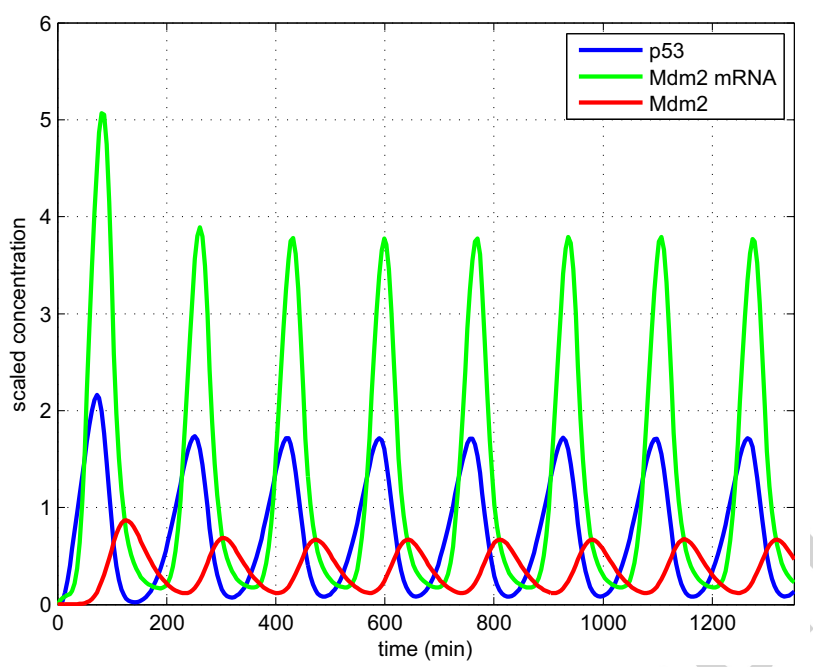

Fig. 17: Plots showing the concentrations of p53 (blue), Mdm2 mRNA (green) and Mdm2 (red) in the nucleus. The period of oscillations is approximately 180 minutes. Parameter values as per (60). 


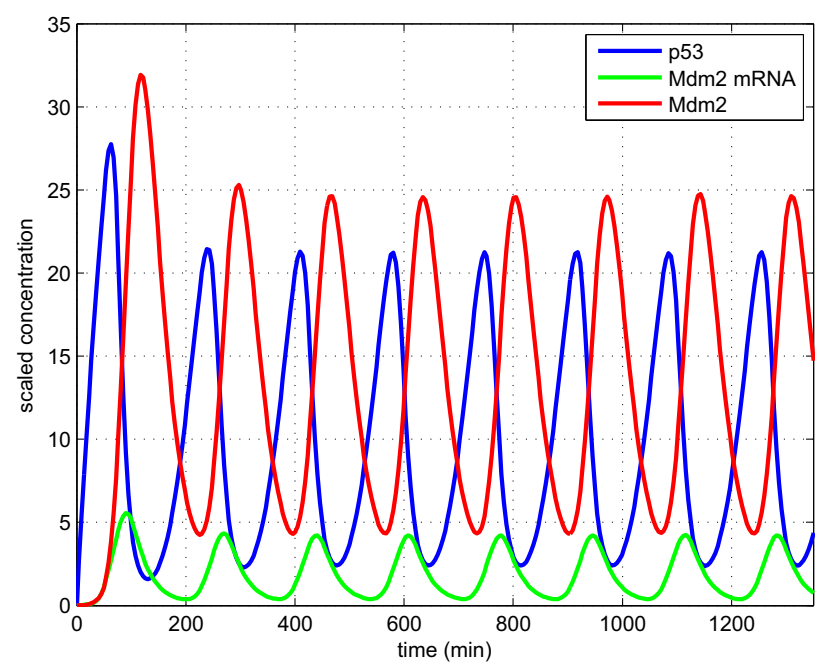

Fig. 18: Plots showing the concentrations of p53 (blue), Mdm2 mRNA (green) and $\mathrm{Mdm} 2$ (red) in the cytoplasm. The period of oscillations is approximately 180 minutes. Parameter values as per (60). 

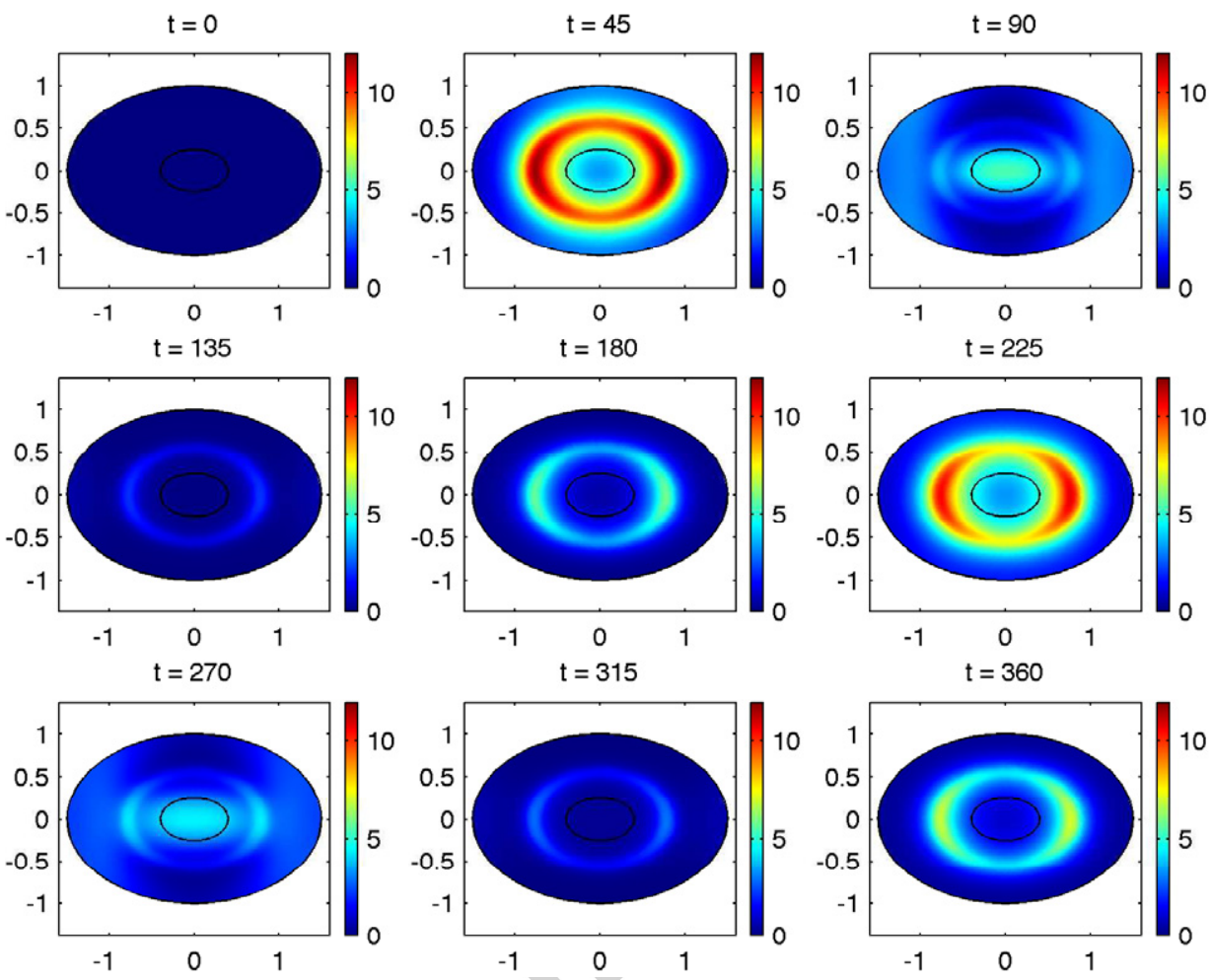

Fig. 19: Plots showing the spatio-temporal evolution of $\mathrm{p} 53$ protein concentration within the cell from times $t=0$ to $t=360$ minutes. The concentration oscillates in both time and space. The patterns of oscillations bear a striking resemblance to those obtained in individual cells in Geva-Zatorsky et al. (2006). Parameter values as per (60). 

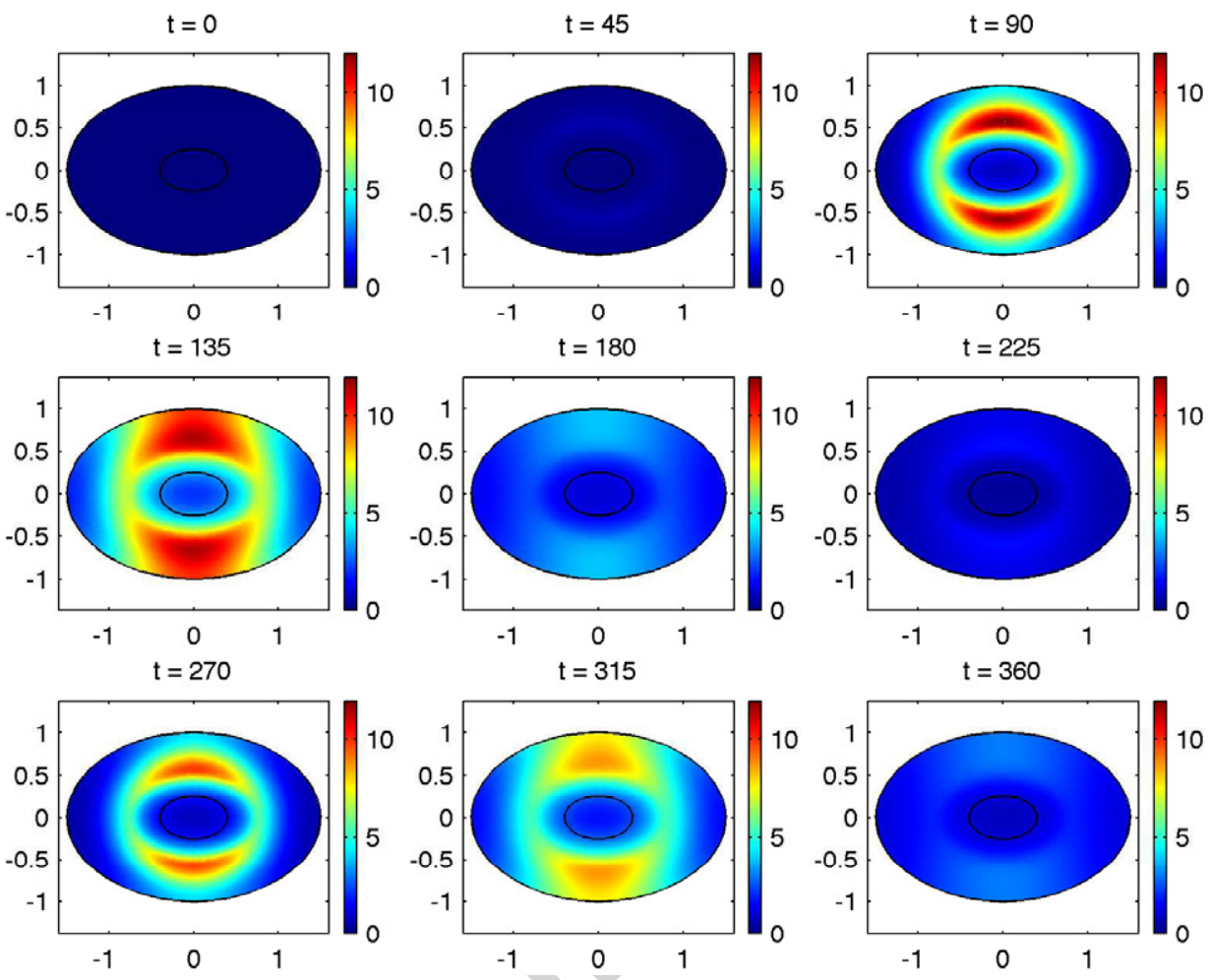

Fig. 20: Plots showing the spatio-temporal evolution of Mdm2 protein concentration within the cell from times $t=0$ to $t=360$ minutes. The concentration oscillates in both time and space. Parameter values as per (60). 


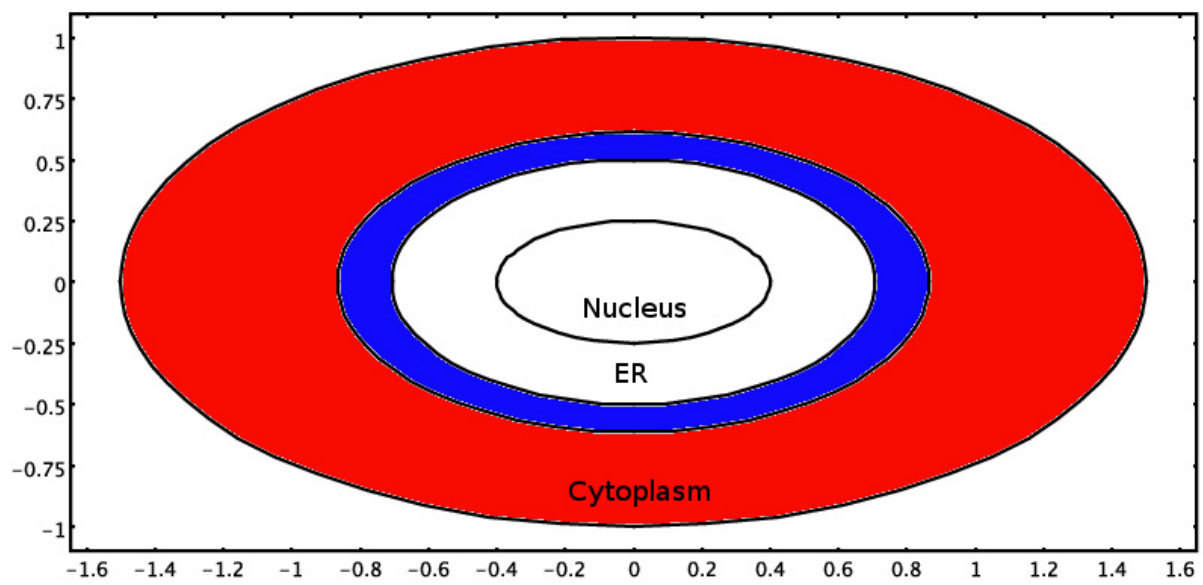

Fig. 21: Schematic diagram showing the regions where the two functions $H_{1}(\bar{x}, \bar{y})$ and $H_{2}(\bar{x}, \bar{y})$ are non-zero. The blue region of the cytoplasm depicts where we allow constant protein synthesis to occur, i.e., this represents the rectangular function $H_{2}(\bar{x}, \bar{y})$. The blue and red regions together depict where we allow protein translation via mRNA, i.e., this represents the function $H_{1}(\bar{x}, \bar{y})$. In the white region representing the ER and nucleus, no protein synthesis takes place. 


\section{Graphical Abstract}

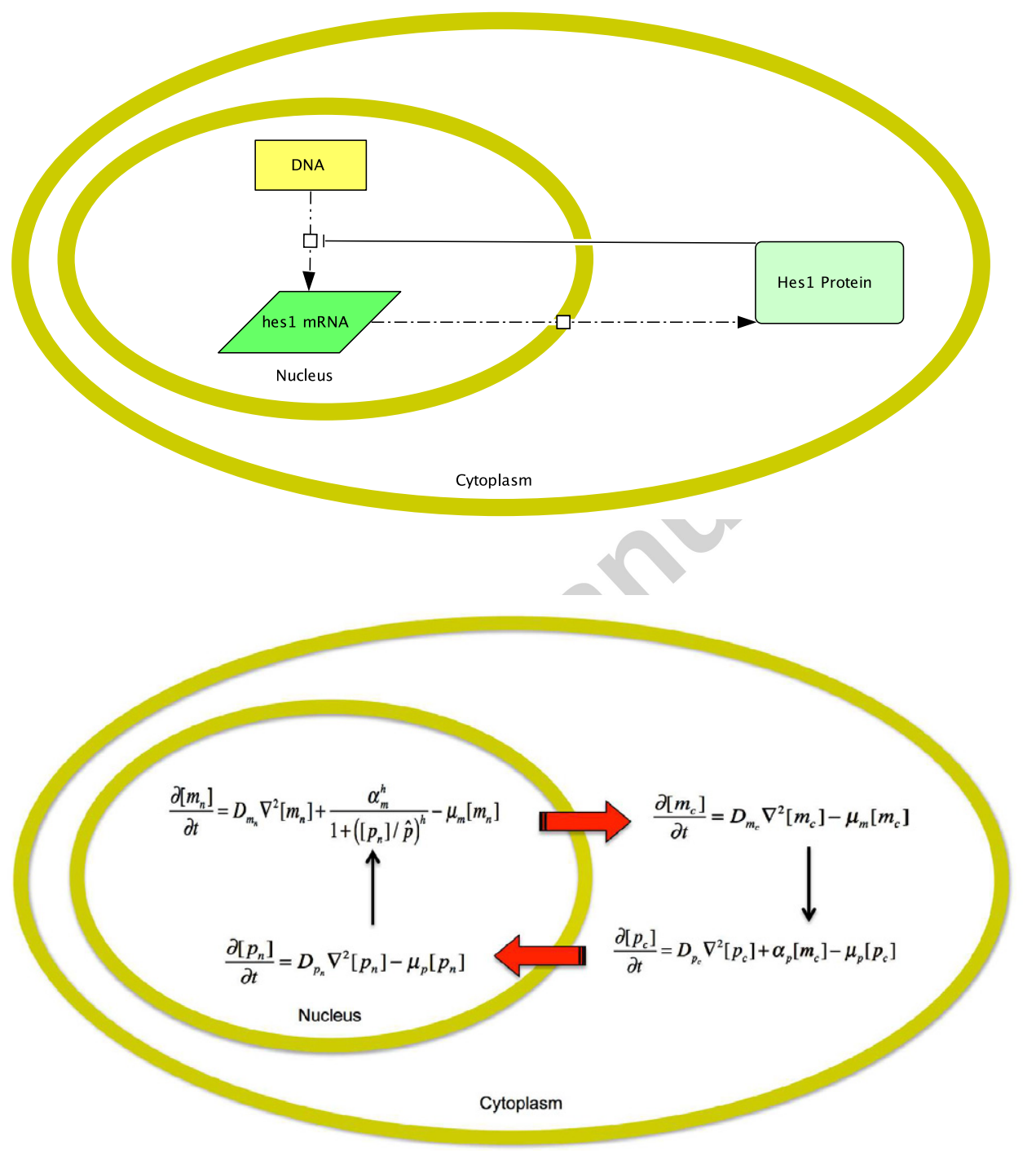

The components of the Hes1 partial differential equation (PDE) system are shown in the schematic diagram above. hes1 mRNA $\left(m_{n}\right)$ is produced in the nucleus, from where it is exported to the cytoplasm $\left(m_{c}\right)$. Here the process of translation takes place, creating Hes1 protein $\left(p_{c}\right)$. Hes1 protein then translocates to the nucleus $\left(p_{n}\right)$ where it inhibits the production of its own mRNA. 


\section{Research Highlights}

- PDE models of feedback inhibition loops mark a conceptual advance in modelling

- The precise spatial localisation of transcription factors can be determined

- The models yield oscillatory dynamics with a period in line with experimental data

- Computational simulations predict a range for the protein diffusion coefficients 